\title{
Study of Vector Boson Scattering and Search for New Physics in Events with Two Same-Sign Leptons and Two Jets
}

\begin{abstract}
V. Khachatryan et al."
(CMS Collaboration)

(Received 23 October 2014; revised manuscript received 11 December 2014; published 2 February 2015)

A study of vector boson scattering in $p p$ collisions at a center-of-mass energy of $8 \mathrm{TeV}$ is presented. The data sample corresponds to an integrated luminosity of $19.4 \mathrm{fb}^{-1}$ collected with the CMS detector. Candidate events are selected with exactly two leptons of the same charge, two jets with large rapidity separation and high dijet mass, and moderate missing transverse energy. The signal region is expected to be dominated by electroweak same-sign $W$-boson pair production. The observation agrees with the standard model prediction. The observed significance is 2.0 standard deviations, where a significance of 3.1 standard deviations is expected based on the standard model. Cross section measurements for $W^{ \pm} W^{ \pm}$and $W Z$ processes in the fiducial region are reported. Bounds on the structure of quartic vector-boson interactions are given in the framework of dimension-eight effective field theory operators, as well as limits on the production of doubly charged Higgs bosons.
\end{abstract}

DOI: 10.1103/PhysRevLett.114.051801

Vector boson scattering (VBS) and quartic boson couplings are features of the standard model (SM) that remain largely unexplored by the LHC experiments. The observation of a Higgs boson [1-3], in accordance with a key prediction of the SM, motivates further study of the mechanism of electroweak symmetry breaking through measurements of VBS processes. In the absence of the SM Higgs boson, the amplitudes for these processes would increase as a function of center-of-mass energy and ultimately violate unitarity $[4,5]$. The Higgs boson actually observed by the LHC experiments may restore the unitarity, although some scenarios of physics beyond the SM predict enhancements for VBS through modifications to the Higgs sector or the presence of additional resonances [6,7].

This Letter presents a study of VBS in $p p$ collisions at $\sqrt{s}=8 \mathrm{TeV}$. The data sample corresponds to an integrated luminosity of $19.4 \pm 0.5 \mathrm{fb}^{-1}$ collected with the CMS detector [8] at the LHC in 2012. The aim of the analysis is to find evidence for the electroweak production of same-sign $W$-boson pair events. The strong production cross section is reduced by the same-sign requirement, making the experimental signature of same-sign dilepton events with two jets an ideal topology for VBS studies. Candidate events have exactly two identified leptons of the same charge, two jets with large rapidity separation and dijet mass, and moderate missing transverse energy. The final states considered are $\mu^{+} \mu^{+} \nu_{\mu} \nu_{\mu} j j, e^{+} e^{+} \nu_{e} \nu_{e} j j$, $e^{+} \mu^{+} \nu_{e} \nu_{\mu} j$, and their charge conjugates and $\tau$-lepton

* Full author list given at the end of the article.

Published by the American Physical Society under the terms of the Creative Commons Attribution 3.0 License. Further distribution of this work must maintain attribution to the author(s) and the published articles title, journal citation, and DOI.
PACS numbers: 14.70.Fm, 12.60.Cn, 13.38.-b, 13.85.Qk

decays to electrons and muons. Figure 1 shows representative Feynman diagrams for the electroweak and QCD induced production.

The study of VBS presented here leads to measurements of the production cross sections for $W^{ \pm} W^{ \pm}$and $W Z$ in a fiducial region. Evidence for electroweak production has been reported by the ATLAS Collaboration [9]. Various extensions of the SM alter the couplings of vector bosons. An excess of events could signal the presence of anomalous quartic gauge couplings (AQGCs) [10]. Doubly charged Higgs bosons are predicted in Higgs sectors beyond the SM where weak isotriplet scalars are included [11,12]; they can be produced via weak vector-boson fusion (VBF) and decay to pairs of same-sign $W$ bosons [13].
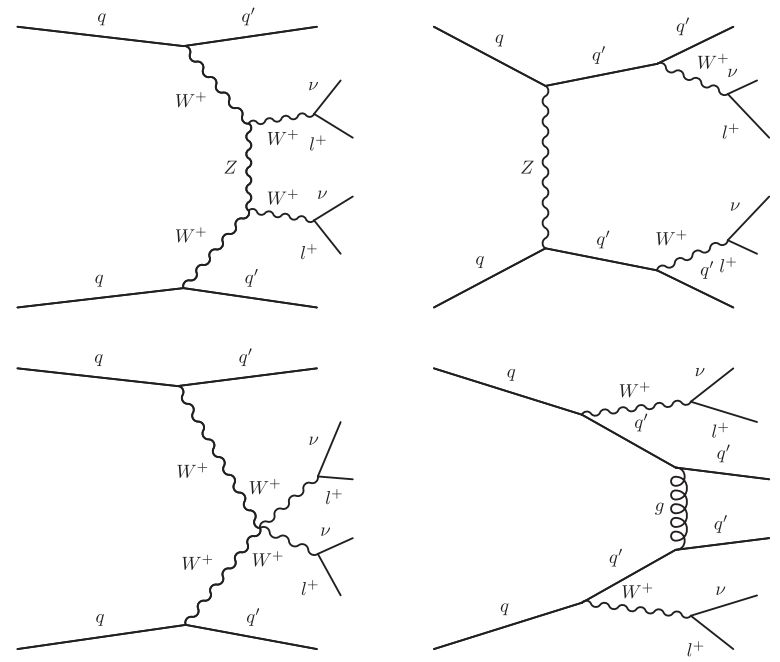

FIG. 1. Representative Feynman diagrams for the electroweak and QCD induced same-sign $W$-boson pair production. 
The central feature of the CMS apparatus is a superconducting solenoid, of $6 \mathrm{~m}$ internal diameter, providing a magnetic field of $3.8 \mathrm{~T}$. Within the field volume are a silicon pixel and strip tracker, a crystal electromagnetic calorimeter, and a brass or scintillator hadron calorimeter. Muons are measured in gas-ionization detectors embedded in the steel flux-return yoke of the magnet. The first level of the CMS trigger system, composed of custom hardware processors, is designed to select the most interesting events within $3 \mu \mathrm{s}$, using information from the calorimeters and muon detectors. The high level trigger processor farm further reduces the event rate to a few hundred hertz before data storage. Details of the CMS detector and its performance can be found elsewhere [8].

Several Monte Carlo (MC) event generators are used to simulate the signal and background processes. The leadingorder event generator MADGRAPH 5.2 [14] is used to produce event samples of diboson production via diagrams with two or fewer powers of $\alpha_{s}$ and up to six electroweak vertices. This includes two categories of diagrams: those with exactly two powers of $\alpha_{s}$ which we refer to as quantum chromodynamic (QCD) production and those with no powers of $\alpha_{s}$, which we refer to as electroweak (EW) production. The EW category includes diagrams with $W W W W$ quartic interactions and diagrams where two same-sign $W$ bosons scatter through the exchange of a Higgs boson, a $Z$ boson, or a photon. Double-parton scattering, triboson production, and doubly charged Higgs boson production samples are also generated using MADGRAPH 5.2. Top-quark background processes are generated with the next-to-leading-order event generator POWHEG 1.0 [15-18]. The set of parton distribution functions (PDFs) used is CTEQ6L [19] for MADGRAPH and CT10 [20] for POWHEG. All event generators are interfaced to PYTHIA 6.4 [21] for the showering of the partons and subsequent hadronization. The PYTHIA parameters for the underlying event were set according to the $Z 2^{*}$ tune [22]. The detector response is simulated by the GEANT4 package [23] using a detailed description of the CMS detector. The average number of simultaneous proton-proton interactions per bunch crossing in the $8 \mathrm{TeV}$ data is approximately 21 ; additional $p p$ interactions overlapping with the event of interest are included in the simulated samples. Collision events are selected by the trigger system requiring the presence of one or two high transverse momentum $\left(p_{T}\right)$ muons or electrons. The trigger efficiency is greater than $99 \%$ for events that pass all other selection criteria explained below. A particle-flow algorithm $[24,25]$ is used to reconstruct all observable particles in the event. It combines all the subdetector information to reconstruct individual particles and identify them as charged hadrons, neutral hadrons, photons, and leptons. The missing transverse energy $E_{T}^{\text {miss }}$ is defined as the magnitude of the negative vector sum of the transverse momenta of all reconstructed particles (charged and neutral) in the event.
The selection of events aims to single out same-sign dilepton events with the VBS topology while reducing the top quark, Drell-Yan, and $W Z$ background processes. All objects are selected following the methods described in Ref. [26]. To avoid bias, the number of events passing the selection was not evaluated until the analysis was complete. Two same-sign lepton candidates, muons or electrons, with $p_{T}>20 \mathrm{GeV}$ and $|\eta|<2.4(2.5)$ for muons (electrons) are required to be isolated from other reconstructed particles in a cone of $\Delta R=0.3$, where $\Delta R=\sqrt{\Delta \eta^{2}+\Delta \phi^{2}}$. Jets are reconstructed using the anti- $k_{T}$ clustering algorithm [27] with a distance parameter $R=0.5$, as implemented in the FASTJET package [28,29]. Events are required to have at least two selected jets with $E_{T}>30 \mathrm{GeV}$ and $|\eta|<4.7$. The VBS topology is targeted by requiring that the two jets with leading $p_{T}$ have large dijet mass, $m_{j j}>500 \mathrm{GeV}$, and large pseudorapidity separation, $\left|\Delta \eta_{j j}\right|>2.5$.

To suppress top-quark backgrounds ( $t \bar{t}$ and $t W$ ), a topquark veto technique is used; it is based on the presence of a soft muon in the event from the semileptonic decay of the bottom quark and on bottom-quark jet tagging criteria based on the impact parameters of the constituent tracks [30]. A minimum dilepton mass, $m_{\ell \ell}>50 \mathrm{GeV}$, is required to reduce the $W+$ jets and top-quark background processes. To reduce the background from $W Z$ production, events with a third, loosely identified lepton with $p_{T}>$ $10 \mathrm{GeV}$ are rejected. Drell-Yan events can be selected if the charge of one lepton is measured incorrectly. To reduce this background, $\left|m_{\ell \ell}-m_{Z}\right|>15 \mathrm{GeV}$ is required for $e^{ \pm} e^{ \pm}$ events. The charge confusion in dimuon events is negligible. The Drell-Yan background is further reduced by requiring $E_{T}^{\text {miss }}>40 \mathrm{GeV}$.

The nonprompt lepton background originating from leptonic decays of heavy quarks, hadrons misidentified as leptons, and electrons from photon conversions, is suppressed by the identification and isolation requirements imposed on muons and electrons. The remaining contribution from the nonprompt lepton background is estimated directly from data. The efficiency for a predefined loose leptonlike object to pass the full lepton selection, typically called the "tight-to-loose ratio" $\left(R_{\mathrm{TL}}\right)$, is estimated in a control sample with one additional lepton candidate that passes the standard lepton selection criteria. To account for the dependence on kinematic observables, this ratio is parameterized as a function of $p_{T}$ and $\eta$. Systematic uncertainties are obtained by the application of $R_{\mathrm{TL}}$ to other control samples, accounting for the sample dependence in the estimation of $R_{\mathrm{TL}}$. The $W Z \rightarrow 3 \ell \nu$ process is normalized in a data control region by requiring a third fully identified lepton with $p_{T}>10 \mathrm{GeV}$. The contribution of opposite-sign dilepton events to the signal region is estimated by applying data-to-simulation charge misidentification scale factors to simulated events with two opposite-sign leptons. The charge-misidentification fraction is estimated using $Z$ boson events and is found to be between 
TABLE I. Signal and background yields after the full selection. Only statistical uncertainties are reported. The signal, $W^{ \pm} W^{ \pm} j j$, includes EW and QCD processes and their interference.

\begin{tabular}{lcccccccr}
\hline \hline & Nonprompt & $W Z$ & $V V V$ & Wrong sign & $W W$ DPS & Total bkg. & $W^{ \pm} W^{ \pm} j j$ & Data \\
\hline$W^{+} W^{+}$ & $2.1 \pm 0.6$ & $0.6 \pm 0.1$ & $0.2 \pm 0.1$ & $0.1 \pm 0.1$ & $0.1 \pm 0.1$ & $3.1 \pm 0.6$ & $7.1 \pm 0.1$ & 10 \\
$W^{-} W^{-}$ & $2.1 \pm 0.5$ & $0.4 \pm 0.1$ & $0.1 \pm 0.1$ & $\ldots$ & $\ldots$ & $2.6 \pm 0.5$ & $1.8 \pm 0.1$ & 2 \\
$W^{ \pm} W^{ \pm}$ & $4.2 \pm 0.8$ & $1.0 \pm 0.1$ & $0.3 \pm 0.1$ & $0.1 \pm 0.1$ & $0.1 \pm 0.1$ & $5.7 \pm 0.8$ & $8.9 \pm 0.1$ & 12 \\
\hline \hline
\end{tabular}

$0.1 \%$ and $0.3 \%$ for electrons, while it is negligible for muons.

After the full selection, about $15 \%$ of the background is due to the $W Z \rightarrow 3 \ell \nu$ process and about $75 \%$ to nonprompt leptons. Backgrounds from opposite-sign lepton pairs misreconstructed as same-sign ("wrong-sign background"), $W W$ production via double parton scattering (DPS), and triboson production $(V V V)$, which includes top-pair plus boson processes, contribute less than $10 \%$.

The expected signal and background yields are shown in Table I for positive and negative pairs separately and for their sum. The signal corresponds to $W^{ \pm} W^{ \pm}$production, including EW and QCD contributions, and their interference, which amounts to approximately 10\%. The EW processes constitute $85 \%-90 \%$ of the total signal contribution. The $m_{j j}$ and leading-lepton $p_{T}$ distributions for the signal and background processes are shown in Fig. 2. In order to quantify the significance of the observation of the production via VBS, a statistical analysis of the event yields is performed in eight bins: four bins in $m_{j j}$ with two bins in the lepton charge.

The signal efficiencies are estimated using simulated samples. In the statistical analysis, shape and normalization uncertainties are considered. The shape uncertainties are estimated by remaking the distribution of a given observable after considering the systematic variations for each source of uncertainty. The lepton trigger, reconstruction, and selection efficiencies are measured using $Z / \gamma^{*} \rightarrow$ $\ell^{+} \ell^{-}$events that provide an unbiased sample with high purity. The estimated uncertainty is $2 \%$ per lepton. The uncertainties due to the momentum scale for electrons and muons are also taken into account and contribute $2 \%$. The jet energy scale and resolution uncertainties give rise to an uncertainty in the yields of about $5 \%$. The uncertainty in the event selection efficiency for events with neutrinos yielding genuine $E_{T}^{\text {miss }}$ in the final state is assessed and leads to an uncertainty of $2 \%$. The uncertainty in the estimated event yields, which is related to the top-quark veto, is evaluated by using a $Z / \gamma^{*} \rightarrow \ell^{+} \ell^{-}$sample with at least two reconstructed jets and is found to be about $2 \%$. The statistical uncertainty in the yield of each bin and for each process is also taken into account. The uncertainty of $2.6 \%$ in the integrated luminosity [31] is considered for all simulated processes. The normalization of the processes with misidentified leptons has a $36 \%$ systematic uncertainty [26], which has two sources: the dependence on the sample composition and the method used to estimate it. The $W Z$ normalization uncertainty is $35 \%$, dominated by the small number of events in the trilepton control region. Theoretical uncertainties are estimated by varying the
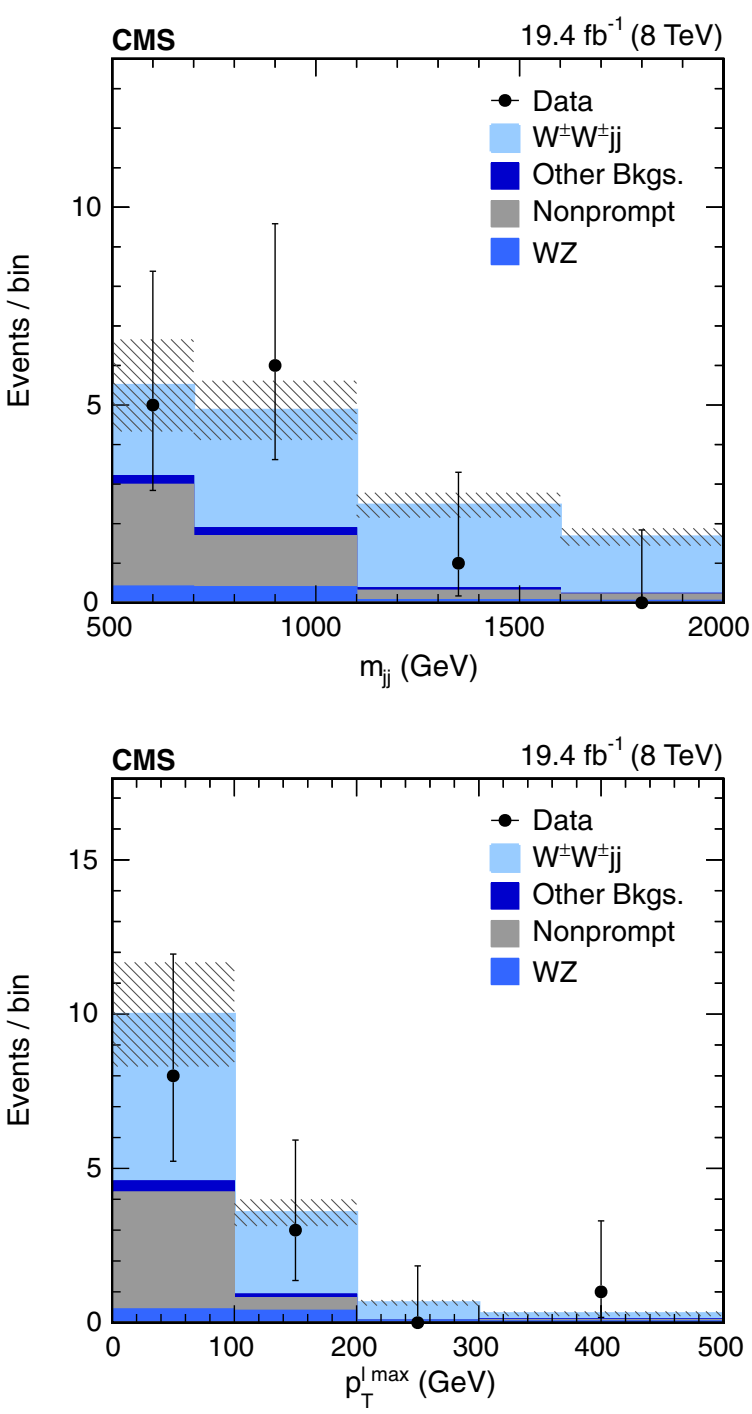

FIG. 2 (color online). The distributions of $m_{j j}$ (top) and leading lepton $p_{T}, p_{T}^{\ell, \max }$, in the signal region (bottom). The hatched bars include statistical and systematic uncertainties. The $W^{+} W^{+}$and $W^{-} W^{-}$candidates are combined in these distributions. The signal, $W^{ \pm} W^{ \pm} j j$, includes EW and QCD processes and their interference. The histograms for other backgrounds include the contributions from wrong-sign events, DPS, and $V V V$ processes. 
renormalization and factorization scales up and down by a factor of two from their nominal value in the event, and found to be $5 \%$ for the signal normalization and $50 \%$ for the triboson background normalization. A PDF uncertainty of $6 \%-8 \%$ in the normalization of the signal and $W Z$ processes is included. The systematic uncertainties of the background normalizations are taken into account using log-normal distributions.

The cross section is extracted for a fiducial signal region. The fiducial region is defined by requiring two same-sign leptons with $p_{T}{ }^{\ell}>10 \mathrm{GeV}$ and $\left|\eta_{\ell}\right|<2.5$, two jets with $p_{T}^{j}>20 \mathrm{GeV}$ and $\left|\eta^{j}\right|<5.0, m_{j j}>300 \mathrm{GeV}$, and $\left|\Delta \eta_{j j}\right|>2.5$ and is less stringent than the event selection for our signal region. The measured cross section is corrected for the acceptance in this region using the MADGRAPH MC generator, which is also used to estimate the theoretical cross section. The acceptance ratio between the selected signal region and the fiducial region is $36 \%$ considering generator-level jet and lepton properties only. The overall acceptance times efficiency is $7.9 \%$.

The MADGRAPH prediction of the same-sign $W$-boson pair cross section is corrected by a next-to-leading order to leading-order cross section ratio estimated using VBFNLO [32-34]. The fiducial cross section is found to be $\sigma_{\text {fid }}\left(W^{ \pm} W^{ \pm} j j\right)=4.0_{-2.0}^{+2.4}$ (stat $)_{-1.0}^{+1.1}$ (syst) fb with an expectation of $5.8 \pm 1.2 \mathrm{fb}$.

In addition to the dilepton same-sign signal region, a $W Z \rightarrow 3 \ell \nu$ control region is studied by requiring an additional lepton with $p_{T}$ larger than $10 \mathrm{GeV}$. This control region allows the measurement of a fiducial cross section of the $W Z j j$ process and is $\sigma_{\text {fid }}(W Z j j)=10.8 \pm 4.0$ (stat $) \pm$ 1.3 (syst) fb with an expectation of $14.4 \pm 4.0 \mathrm{fb}$. The fiducial region is defined in the same way as for the $W W$ analysis, but requiring one more lepton with $p_{T}^{\ell}>$ $10 \mathrm{GeV}$ and $\left|\eta_{\ell}\right|<2.5$. The acceptance ratio between the selected signal region and the fiducial region is $20 \%$ considering generator-level jet and lepton properties only. The overall acceptance times efficiency is $3.6 \%$.

To compute the limits and significances, the $\mathrm{CL}_{s}$ [35-37] construction is used. The observed (expected) significance for the $W^{ \pm} W^{ \pm} j j$ process is $2.0 \sigma(3.1 \sigma)$. Considering the QCD component of the $W^{ \pm} W^{ \pm} j j$ events as background and the EW component together with the EW-QCD interference as signal, the observed (expected) signal significance reduces to $1.9 \sigma(2.9 \sigma)$.

Various extensions to the SM alter the couplings between vector bosons. Reference [10] proposes nine independent $C$ - and $P$-conserving dimension-eight effective operators to modify the quartic couplings between the weak gauge bosons. The variable $m_{\ell \ell}$ is more sensitive to AQGCs than $p_{T}^{\ell, \max }, m_{\ell \ell j j}$, and $m_{j j}$. Figure 3 (top) shows the expected $m_{\ell \ell}$ distribution for three values of $F_{T, 0} / \Lambda^{4} ; \Lambda$ is the scale of new physics and $F_{T, 0}$ is the coefficient of one of the nine effective operators. The observed and expected upper and lower limits at $95 \%$ confidence level (C.L.). on the nine
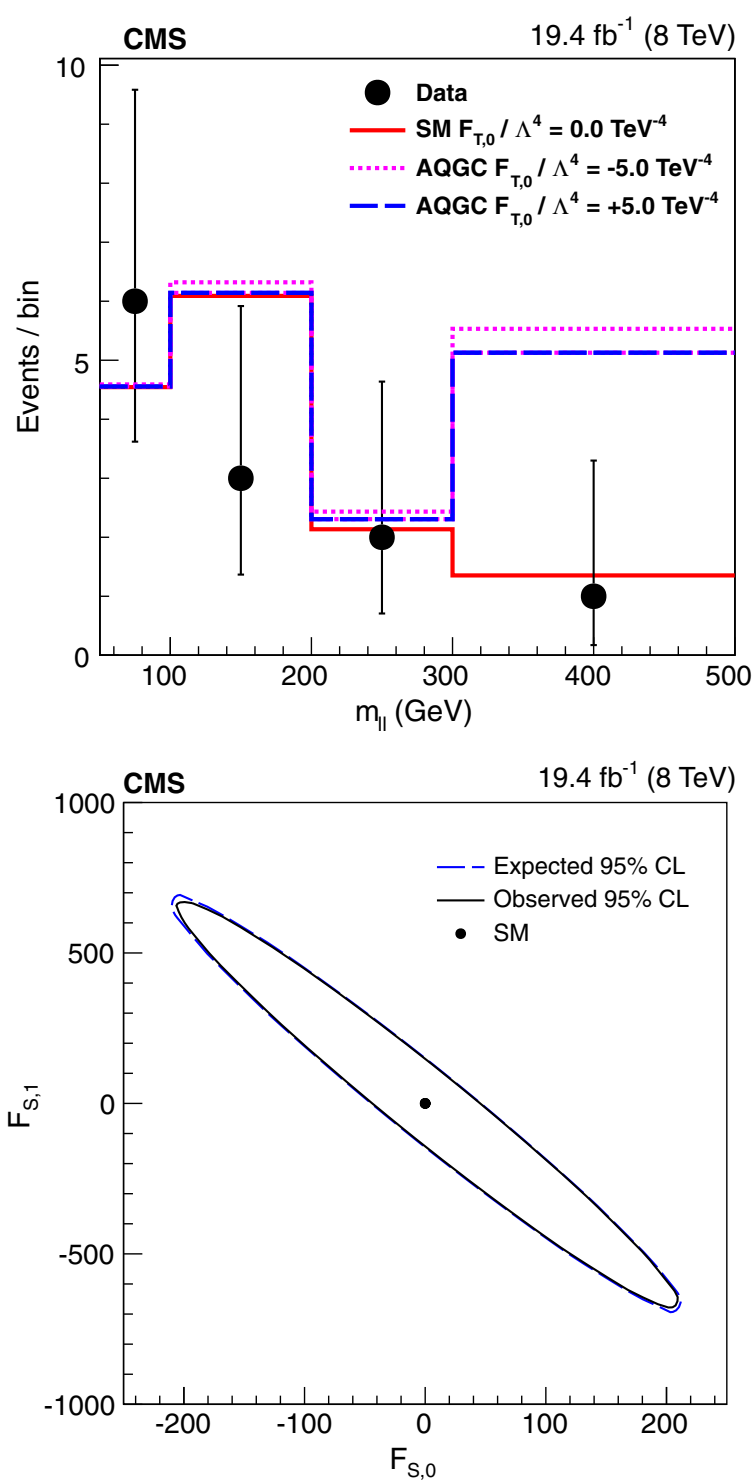

FIG. 3 (color online). The $m_{\ell \ell}$ distributions (top) after full selection with all SM backgrounds and $F_{T, 0} / \Lambda^{4}=-5.0,0$ (SM), and $5.0 \mathrm{TeV}^{-4}$; the last bin includes overflow events. Observed and expected two-dimensional 95\% C.L. (bottom) for $F_{S 0} / \Lambda^{4}$ and $F_{S 1} / \Lambda^{4}$.

coefficients are shown in Table II, where all the results are obtained by varying the effective operators one by one. The effect of possible AQGCs on the $W Z$ process in the signal region is negligible. Some operators for anomalous quartic gauge boson couplings may lead to tree-level unitarity violation. We also report the values of the operator coefficient for which unitarity is restored at the scale of $8 \mathrm{TeV}$, the unitarity limit. In addition to the limits on individual operator coefficients, the expected and observed two-dimensional 95\% C.L. on $F_{S, 0} / \Lambda^{4}$ and $F_{S, 1} / \Lambda^{4}$ are presented in Fig. 3 (bottom): a linear combination of those operators leads to a scaling of the SM cross section.

Doubly charged Higgs bosons are predicted in models that contain a Higgs triplet field. Some of these scenarios 
TABLE II. Observed and expected upper and lower limits at 95\% C.L. on the nine dimension-eight operators that affect quartic couplings between the weak gauge bosons. Limits from unitarity are reported. The units are $\mathrm{TeV}^{-4}$.

\begin{tabular}{lccccc}
\hline \hline $\begin{array}{l}\text { Operator } \\
\text { coefficient }\end{array}$ & $\begin{array}{c}\text { Exp. } \\
\text { lower }\end{array}$ & $\begin{array}{c}\text { Exp. } \\
\text { upper }\end{array}$ & $\begin{array}{c}\text { Obs. } \\
\text { lower }\end{array}$ & $\begin{array}{c}\text { Obs. } \\
\text { upper }\end{array}$ & $\begin{array}{c}\text { Unitarity } \\
\text { limit }\end{array}$ \\
\hline$F_{S, 0} / \Lambda^{4}$ & -42 & 43 & -38 & 40 & 0.016 \\
$F_{S, 1} / \Lambda^{4}$ & -129 & 131 & -118 & 120 & 0.050 \\
$F_{M, 0} / \Lambda^{4}$ & -35 & 35 & -33 & 32 & 80 \\
$F_{M, 1} / \Lambda^{4}$ & -49 & 51 & -44 & 47 & 205 \\
$F_{M, 6} / \Lambda^{4}$ & -70 & 69 & -65 & 63 & 160 \\
$F_{M, 7} / \Lambda^{4}$ & -76 & 73 & -70 & 66 & 105 \\
$F_{T, 0} / \Lambda^{4}$ & -4.6 & 4.9 & -4.2 & 4.6 & 0.027 \\
$F_{T, 1} / \Lambda^{4}$ & -2.1 & 2.4 & -1.9 & 2.2 & 0.022 \\
$F_{T, 2} / \Lambda^{4}$ & -5.9 & 7.0 & -5.2 & 6.4 & 0.08 \\
\hline \hline
\end{tabular}

predict same-sign dilepton events from $W^{ \pm} W^{ \pm}$decays with a VBF topology. The cross section for VBF production of $H^{ \pm \pm}$and decay to $W^{ \pm} W^{ \pm}$is directly proportional to the vacuum expectation value of the triplet. The remaining five parameters in the model of the Higgs potential are adjusted to get the given $m_{H^{ \pm \pm}}$hypothesis while requiring one of the scalar singlets to have a mass of $125 \mathrm{GeV}$. The GeorgiMachacek model of Higgs triplets [38] is considered. For $m_{H^{ \pm \pm}}=200(800) \mathrm{GeV}$ the following parameters are used: $\lambda_{1}=1, \lambda_{2}=1, \lambda_{3}=1, \lambda_{4}=2.37(4)$, and $\lambda_{5}=$ 0.432 (7.26). By using the $m_{j j}$ distribution, 95\% C.L. upper limits on $\sigma_{H^{ \pm \pm}} \mathcal{B}\left(H^{ \pm \pm} \rightarrow W^{ \pm} W^{ \pm}\right)$are derived as shown in Fig. 4. The experimental results are overlaid with theoretical cross sections for three values of the vacuum expectation value.

In summary, a study of vector boson scattering in $p p$ collisions at $\sqrt{s}=8 \mathrm{TeV}$ has been presented based on a

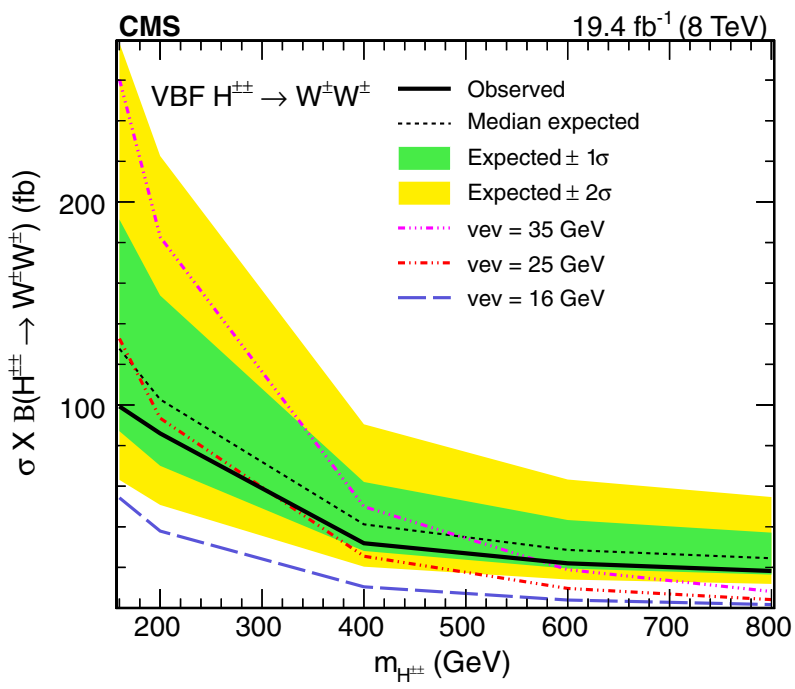

FIG. 4 (color online). Expected and observed 95\% C.L. upper limits on the cross section times branching fraction, $\sigma_{H^{ \pm \pm}} \mathcal{B}\left(H^{ \pm \pm} \rightarrow W^{ \pm} W^{ \pm}\right)$. Theoretical cross sections for three values of the vacuum expectation value (vev) are overlaid. data sample corresponding to an integrated luminosity of $19.4 \mathrm{fb}^{-1}$. Candidate events are selected with exactly two leptons of the same charge, two jets with large rapidity separation and dijet mass, and moderate missing transverse energy. The signal region is expected to be dominated by electroweak same-sign $W$-boson pair production. The observation agrees with the standard model prediction. The observed significance is 2.0 standard deviations, where a significance of 3.1 standard deviations is expected based on the standard model. Cross section measurements for $W^{ \pm} W^{ \pm}$and $W Z$ processes in the fiducial region are reported. Bounds on the structure of quartic vector-boson interactions are given in the framework of dimension-eight effective field theory operators, as well as limits on the production of doubly charged Higgs bosons.

We congratulate our colleagues in the CERN accelerator departments for the excellent performance of the LHC and thank the technical and administrative staffs at CERN and at other CMS institutes for their contributions to the success of the CMS effort. In addition, we gratefully acknowledge the computing centers and personnel of the Worldwide LHC Computing Grid for delivering so effectively the computing infrastructure essential to our analyses. Finally, we acknowledge the enduring support for the construction and operation of the LHC and the CMS detector provided by the following funding agencies: BMWFW and FWF (Austria); FNRS and FWO (Belgium); CNPq, CAPES, FAPERJ, and FAPESP (Brazil); MES (Bulgaria); CERN; CAS, MoST, and NSFC (China); COLCIENCIAS (Colombia); MSES and CSF (Croatia); RPF (Cyprus); MoER, ERC IUT and ERDF (Estonia); Academy of Finland, MEC, and HIP (Finland); CEA and CNRS/IN2P3 (France); BMBF, DFG, and HGF (Germany); GSRT (Greece); OTKA and NIH (Hungary); DAE and DST (India); IPM (Iran); SFI (Ireland); INFN (Italy); NRF and WCU (Republic of Korea); LAS (Lithuania); MOE and UM (Malaysia); CINVESTAV, CONACYT, SEP, and UASLP-FAI (Mexico); MBIE (New Zealand); PAEC (Pakistan); MSHE and NSC (Poland); FCT (Portugal); JINR (Dubna); MON, RosAtom, RAS and RFBR (Russia); MESTD (Serbia); SEIDI and CPAN (Spain); Swiss Funding Agencies (Switzerland); MST (Taipei); ThEPCenter, IPST, STAR and NSTDA (Thailand); TUBITAK and TAEK (Turkey); NASU and SFFR (Ukraine); STFC (United Kingdom); DOE and NSF (USA).

[1] ATLAS Collaboration, Phys. Lett. B 716, 1 (2012).

[2] CMS Collaboration, Phys. Lett. B 716, 30 (2012).

[3] CMS Collaboration, J. High Energy Phys. 06 (2013) 081.

[4] B. W. Lee, C. Quigg, and H. B. Thacker, Phys. Rev. Lett. 38, 883 (1977). 
[5] B. W. Lee, C. Quigg, and H. B. Thacker, Phys. Rev. D 16, 1519 (1977).

[6] D. Espriu and B. Yencho, Phys. Rev. D 87, 055017 (2013).

[7] J. Chang, K. Cheung, C.-T. Lu, and T.-C. Yuan, Phys. Rev. D 87, 093005 (2013).

[8] CMS Collaboration, JINST 3, S08004 (2008).

[9] ATLAS Collaboration, Phys. Rev. Lett. 113, 141803 (2014).

[10] O. J. P. Éboli, M. C. Gonzalez-Garcia, and J. K. Mizukoshi, Phys. Rev. D 74, 073005 (2006).

[11] Ch. Englert, E. Re, and M. Spannowsky, Phys. Rev. D 87, 095014 (2013).

[12] Ch. Englert, E. Re, and M. Spannowsky, Phys. Rev. D 88, 035024 (2013).

[13] Ch.-W. Chiang, T. Nomura, and K. Tsumura, Phys. Rev. D 85, 095023 (2012).

[14] J. Alwall, R. Frederix, S. Frixione, V. Hirschi, F. Maltoni, O. Mattelaer, H.-S. Shao, T. Stelzer, P. Torrielli, and M. Zaro, J. High Energy Phys. 07 (2014) 079.

[15] S. Alioli, P. Nason, C. Oleari, and E. Re, J. High Energy Phys. 07 (2008) 060.

[16] P. Nason, J. High Energy Phys. 11 (2004) 040.

[17] S. Frixione, P. Nason, and C. Oleari, J. High Energy Phys. 11 (2007) 070.

[18] S. Alioli, P. Nason, C. Oleari, and E. Re, J. High Energy Phys. 06 (2010) 043.

[19] H.-L. Lai, J. Huston, Z. Li, P. Nadolsky, J. Pumplin, D. Stump, and C.-P. Yuan, Phys. Rev. D 82, 054021 (2010).

[20] H.-L. Lai, M. Guzzi, J. Huston, Z. Li, P. M. Nadolsky, J. Pumplin, and C.-P. Yuan, Phys. Rev. D 82, 074024 (2010).

[21] T. Sjöstrand, S. Mrenna, and P. Skands, J. High Energy Phys. 05 (2006) 026.

[22] CMS Collaboration, J. High Energy Phys. 09 (2011) 109.
[23] S. Agostinelli et al. (GEANT4 Collaboration), Nucl. Instrum. Methods Phys. Res., Sect. A 506, 250 (2003).

[24] CMS Collaboration, CMS Physics Analysis Summary Report No. CMS-PAS-PFT-09-001, 2009, http://cdsweb .cern.ch/record/1194487.

[25] CMS Collaboration, CMS Physics Analysis Summary Report No. CMS-PAS-PFT-10-002, 2010.

[26] CMS Collaboration, J. High Energy Phys. 01 (2014) 096.

[27] M. Cacciari, G. P. Salam, and G. Soyez, J. High Energy Phys. 04 (2008) 063.

[28] M. Cacciari, G. P. Salam, and G. Soyez, Eur. Phys. J. C 72, 1896 (2012).

[29] M. Cacciari and G. P. Salam, Phys. Lett. B 641, 57 (2006).

[30] CMS Collaboration, JINST 8, P04013 (2012).

[31] CMS Collaboration, CMS Physics Analysis Summary Report No. CMS-PAS-LUM-13-001 2013, http://cdsweb .cern.ch/record/1598864.

[32] J. Baglio, J. Bellm, F. Campanario, B. Feigl, J. Frank, T. Figy, M. Kerner, L. D. Ninh, S. Palmer, M. Rauch, F. Schissler, O. Schlimpert, and D. Zeppenfeld, arXiv: 1404.3940.

[33] J. Baglio et al., arXiv:1107.4038.

[34] K. Arnold et al., Comput. Phys. Commun. 180, 1661 (2009).

[35] A. L. Read, J. Phys. G 28, 2693 (2002).

[36] T. Junk, Nucl. Instrum. Methods Phys. Res., Sect. A 434, 435 (1999).

[37] ATLAS and CMS Collaborations, Tech. Rep No. ATLPHYS-PUB 2011-11, CMS-NOTE 2011-005 2011, http:// cdsweb.cern.ch/record/1379837.

[38] H. Georgi and M. Machacek, Nucl. Phys. B 262, 463 (1985).

V. Khachatryan, ${ }^{1}$ A. M. Sirunyan, ${ }^{1}$ A. Tumasyan, ${ }^{1}$ W. Adam, ${ }^{2}$ T. Bergauer, ${ }^{2}$ M. Dragicevic, ${ }^{2}$ J. Erö, ${ }^{2}$ M. Friedl, ${ }^{2}$ R. Frühwirth, ${ }^{2, b}$ V. M. Ghete, ${ }^{2}$ C. Hartl, ${ }^{2}$ N. Hörmann, ${ }^{2}$ J. Hrubec, ${ }^{2}$ M. Jeitler, ${ }^{2, b}$ W. Kiesenhofer, ${ }^{2}$ V. Knünz, ${ }^{2}$ M. Krammer, ${ }^{2, b}$ I. Krätschmer, ${ }^{2}$ D. Liko, ${ }^{2}$ I. Mikulec, ${ }^{2}$ D. Rabady, ${ }^{2, \mathrm{c}}$ B. Rahbaran, ${ }^{2}$ H. Rohringer, ${ }^{2}$ R. Schöfbeck, ${ }^{2}$ J. Strauss, ${ }^{2}$ W. Treberer-Treberspurg, ${ }^{2}$ W. Waltenberger, ${ }^{2}$ C.-E. Wulz, ${ }^{2, b}$ V. Mossolov, ${ }^{3}$ N. Shumeiko, ${ }^{3}$ J. Suarez Gonzalez, ${ }^{3}$ S. Alderweireldt, ${ }^{4}$ M. Bansal, ${ }^{4}$ S. Bansal, ${ }^{4}$ T. Cornelis, ${ }^{4}$ E. A. De Wolf, ${ }^{4}$ X. Janssen, ${ }^{4}$ A. Knutsson, ${ }^{4}$ J. Lauwers, ${ }^{4}$ S. Luyckx, ${ }^{4}$ S. Ochesanu, ${ }^{4}$ R. Rougny, ${ }^{4}$ M. Van De Klundert, ${ }^{4}$ H. Van Haevermaet, ${ }^{4}$ P. Van Mechelen, ${ }^{4}$ N. Van Remortel, ${ }^{4}$ A. Van Spilbeeck, ${ }^{4}$ F. Blekman, ${ }^{5}$ S. Blyweert, ${ }^{5}$ J. D'Hondt, ${ }^{5}$ N. Daci, ${ }^{5}$ N. Heracleous, ${ }^{5}$ J. Keaveney,,${ }^{5}$ S. Lowette, ${ }^{5}$ M. Maes, ${ }^{5}$ A. Olbrechts, ${ }^{5}$ Q. Python, ${ }^{5}$ D. Strom, ${ }^{5}$ S. Tavernier, ${ }^{5}$ W. Van Doninck,${ }^{5}$ P. Van Mulders, ${ }^{5}$ G. P. Van Onsem, ${ }^{5}$ I. Villella, ${ }^{5}$ C. Caillol, ${ }^{6}$ B. Clerbaux, ${ }^{6}$ G. De Lentdecker, ${ }^{6}$ D. Dobur, ${ }^{6}$ L. Favart, ${ }^{6}$ A. P. R. Gay, ${ }^{6}$ A. Grebenyuk, ${ }^{6}$ A. Léonard ${ }^{6}$ A. Mohammadi, ${ }^{6}$ L. Pernie ${ }^{6, c}$ T. Reis, ${ }^{6}$ T. Seva, ${ }^{6}$ L. Thomas, ${ }^{6}$ C. Vander Velde, ${ }^{6}$ P. Vanlaer, ${ }^{6}$ J. Wang, ${ }^{6}$ F. Zenoni, ${ }^{6}$ V. Adler, ${ }^{7}$ K. Beernaert, ${ }^{7}$ L. Benucci, ${ }^{7}$ A. Cimmino, ${ }^{7}$ S. Costantini, ${ }^{7}$ S. Crucy, ${ }^{7}$ S. Dildick, ${ }^{7}$ A. Fagot, ${ }^{7}$ G. Garcia, ${ }^{7}$ J. Mccartin, ${ }^{7}$ A. A. Ocampo Rios, ${ }^{7}$ D. Ryckbosch, ${ }^{7}$ S. Salva Diblen, ${ }^{7}$ M. Sigamani, ${ }^{7}$ N. Strobbe, ${ }^{7}$ F. Thyssen, ${ }^{7}$ M. Tytgat, ${ }^{7}$ E. Yazgan, ${ }^{7}$ N. Zaganidis, ${ }^{7}$ S. Basegmez, ${ }^{8}$ C. Beluffi, ${ }^{8, d}$ G. Bruno, ${ }^{8}$ R. Castello, ${ }^{8}$ A. Caudron,${ }^{8}$ L. Ceard,${ }^{8}$ G. G. Da Silveira,${ }^{8}$ C. Delaere, ${ }^{8}$ T. du Pree, ${ }^{8}$ D. Favart, ${ }^{8}$ L. Forthomme, ${ }^{8}$ A. Giammanco, ${ }^{8, e}$ J. Hollar, ${ }^{8}$ A. Jafari, ${ }^{8}$ P. Jez, ${ }^{8}$ M. Komm, ${ }^{8}$ V. Lemaitre, ${ }^{8}$ C. Nuttens, ${ }^{8}$ D. Pagano, ${ }^{8}$ L. Perrini,${ }^{8}$ A. Pin,${ }^{8}$ K. Piotrzkowski, ${ }^{8}$ A. Popov,${ }^{8, f}$ L. Quertenmont, ${ }^{8}$ M. Selvaggi, ${ }^{8}$ M. Vidal Marono, ${ }^{8}$ J. M. Vizan Garcia, ${ }^{8}$ N. Beliy, ${ }^{9}$ T. Caebergs, ${ }^{9}$ E. Daubie, ${ }^{9}$ G. H. Hammad, ${ }^{9}$ W. L. Aldá Júnior, ${ }^{10}$ G. A. Alves,${ }^{10}$ L. Brito, ${ }^{10}$ M. Correa Martins Junior, ${ }^{10}$ T. Dos Reis Martins, ${ }^{10}$ C. Mora Herrera, ${ }^{10}$ M. E. Pol,,${ }^{10}$ W. Carvalho, ${ }^{11}$ J. Chinellato, ${ }^{11, \mathrm{~g}}$ A. Custódio, ${ }^{11}$ E. M. Da Costa, ${ }^{11}$ D. De Jesus Damiao, ${ }^{11}$ C. De Oliveira Martins,${ }^{11}$ S. Fonseca De Souza, ${ }^{11}$ H. Malbouisson, ${ }^{11}$ D. Matos Figueiredo, ${ }^{11}$ L. Mundim, ${ }^{11}$ H. Nogima, ${ }^{11}$ W. L. Prado Da Silva, ${ }^{11}$ J. Santaolalla, ${ }^{11}$ A. Santoro, ${ }^{11}$ A. Sznajder, ${ }^{11}$ E. J. Tonelli Manganote, ${ }^{11, g}$ A. Vilela Pereira, ${ }^{11}$ C. A. Bernardes ${ }^{12 b}$ S. Dogra ${ }^{12 a}$ 
T. R. Fernandez Perez Tomei, ${ }^{12 a}$ E. M. Gregores, ${ }^{12 b}$ P. G. Mercadante, ${ }^{12 b}$ S. F. Novaes, ${ }^{12 a}$ Sandra S. Padula, ${ }^{12 a}$ A. Aleksandrov,${ }^{13}$ V. Genchev, ${ }^{13, c}$ P. Iaydjiev, ${ }^{13}$ A. Marinov, ${ }^{13}$ S. Piperov,${ }^{13}$ M. Rodozov, ${ }^{13}$ G. Sultanov,${ }^{13}$ M. Vutova, ${ }^{13}$ A. Dimitrov, ${ }^{14}$ I. Glushkov, ${ }^{14}$ R. Hadjiiska, ${ }^{14}$ L. Litov, ${ }^{14}$ B. Pavlov, ${ }^{14}$ P. Petkov, ${ }^{14}$ J. G. Bian, ${ }^{15}$ G. M. Chen, ${ }^{15}$ H. S. Chen, ${ }^{15}$ M. Chen, ${ }^{15}$ T. Cheng, ${ }^{15}$ R. Du, ${ }^{15}$ C. H. Jiang, ${ }^{15}$ R. Plestina, ${ }^{15, h}$ F. Romeo, ${ }^{15}$ J. Tao, ${ }^{15}$ Z. Wang, ${ }^{15}$ C. Asawatangtrakuldee, ${ }^{16}$ Y. Ban, ${ }^{16}$ Q. Li, ${ }^{16}$ S. Liu, ${ }^{16}$ Y. Mao, ${ }^{16}$ S. J. Qian, ${ }^{16}$ D. Wang, ${ }^{16}$ W. Zou, ${ }^{16}$ C. Avila, ${ }^{17}$ A. Cabrera,${ }^{17}$ L. F. Chaparro Sierra,${ }^{17}$ C. Florez ${ }^{17}$ J. P. Gomez, ${ }^{17}$ B. Gomez Moreno, ${ }^{17}$ J. C. Sanabria,${ }^{17}$ N. Godinovic,${ }^{18}$ D. Lelas, ${ }^{18}$ D. Polic, ${ }^{18}$ I. Puljak, ${ }^{18}$ Z. Antunovic, ${ }^{19}$ M. Kovac, ${ }^{19}$ V. Brigljevic, ${ }^{20}$ K. Kadija,${ }^{20}$ J. Luetic, ${ }^{20}$ D. Mekterovic ${ }^{20}$ L. Sudic,${ }^{20}$ A. Attikis,${ }^{21}$ G. Mavromanolakis, ${ }^{21}$ J. Mousa, ${ }^{21}$ C. Nicolaou, ${ }^{21}$ F. Ptochos,${ }^{21}$ P. A. Razis, ${ }^{21}$ M. Bodlak, ${ }^{22}$ M. Finger, ${ }^{22}$ M. Finger Jr., ${ }^{22, i}$ Y. Assran, ${ }^{23, j}$ A. Ellithi Kamel, ${ }^{23, k}$ M. A. Mahmoud ${ }^{23,1}$ A. Radi, ${ }^{23, m, n}$ M. Kadastik, ${ }^{24}$ M. Murumaa, ${ }^{24}$ M. Raidal, ${ }^{24}$ A. Tiko, ${ }^{24}$ P. Eerola, ${ }^{25}$ G. Fedi, ${ }^{25}$ M. Voutilainen, ${ }^{25}$ J. Härkönen, ${ }^{26}$ V. Karimäki, ${ }^{26}$ R. Kinnunen, ${ }^{26}$ M. J. Kortelainen, ${ }^{26}$ T. Lampén, ${ }^{26}$ K. Lassila-Perini, ${ }^{26}$ S. Lehti, ${ }^{26}$ T. Lindén, ${ }^{26}$ P. Luukka, ${ }^{26}$ T. Mäenpää, ${ }^{26}$ T. Peltola, ${ }^{26}$ E. Tuominen, ${ }^{26}$ J. Tuominiemi, ${ }^{26}$ E. Tuovinen, ${ }^{26}$ L. Wendland, ${ }^{26}$ J. Talvitie, ${ }^{27}$ T. Tuuva, ${ }^{27}$ M. Besancon, ${ }^{28}$ F. Couderc, ${ }^{28}$ M. Dejardin, ${ }^{28}$ D. Denegri, ${ }^{28}$ B. Fabbro, ${ }^{28}$ J. L. Faure, ${ }^{28}$ C. Favaro, ${ }^{28}$ F. Ferri, ${ }^{28}$ S. Ganjour, ${ }^{28}$ A. Givernaud ${ }^{28}$ P. Gras, ${ }^{28}$ G. Hamel de Monchenault,${ }^{28}$ P. Jarry, ${ }^{28}$ E. Locci, ${ }^{28}$ J. Malcles, ${ }^{28}$ J. Rander, ${ }^{28}$ A. Rosowsky, ${ }^{28}$ M. Titov,${ }^{28}$ S. Baffioni, ${ }^{29}$ F. Beaudette, ${ }^{29}$ P. Busson, ${ }^{29}$ C. Charlot ${ }^{29}$ T. Dahms,${ }^{29}$ M. Dalchenko, ${ }^{29}$ L. Dobrzynski, ${ }^{29}$ N. Filipovic, ${ }^{29}$ A. Florent, ${ }^{29}$ R. Granier de Cassagnac, ${ }^{29}$ L. Mastrolorenzo, ${ }^{29}$ P. Miné, ${ }^{29}$ C. Mironov, ${ }^{29}$ I. N. Naranjo, ${ }^{29}$ M. Nguyen, ${ }^{29}$ C. Ochando,${ }^{29}$ P. Paganini, ${ }^{29}$ S. Regnard, ${ }^{29}$ R. Salerno, ${ }^{29}$ J. B. Sauvan, ${ }^{29}$ Y. Sirois,${ }^{29}$ C. Veelken, ${ }^{29}$ Y. Yilmaz,${ }^{29}$ A. Zabi, ${ }^{29}$ J.-L. Agram,,${ }^{30,0}$ J. Andrea,${ }^{30}$ A. Aubin, ${ }^{30}$ D. Bloch, ${ }^{30}$ J.-M. Brom, ${ }^{30}$ E. C. Chabert, ${ }^{30}$ C. Collard ${ }^{30}$ E. Conte, ${ }^{30, o}$ J.-C. Fontaine, ${ }^{30, o}$ D. Gelé, ${ }^{30}$ U. Goerlach, ${ }^{30}$

C. Goetzmann, ${ }^{30}$ A.-C. Le Bihan, ${ }^{30}$ P. Van Hove ${ }^{30}$ S. Gadrat, ${ }^{31}$ S. Beauceron, ${ }^{32}$ N. Beaupere, ${ }^{32}$ G. Boudoul,,${ }^{32, c}$

E. Bouvier, ${ }^{32}$ S. Brochet, ${ }^{32}$ C. A. Carrillo Montoya, ${ }^{32}$ J. Chasserat,${ }^{32}$ R. Chierici, ${ }^{32}$ D. Contardo, ${ }^{32, c}$ P. Depasse, ${ }^{32}$ H. El Mamouni, ${ }^{32}$ J. Fan, ${ }^{32}$ J. Fay, ${ }^{32}$ S. Gascon, ${ }^{32}$ M. Gouzevitch, ${ }^{32}$ B. Ille, ${ }^{32}$ T. Kurca, ${ }^{32}$ M. Lethuillier, ${ }^{32}$ L. Mirabito, ${ }^{32}$ S. Perries,${ }^{32}$ J. D. Ruiz Alvarez, ${ }^{32}$ D. Sabes, ${ }^{32}$ L. Sgandurra ${ }^{32}$ V. Sordini, ${ }^{32}$ M. Vander Donckt, ${ }^{32}$ P. Verdier, ${ }^{32}$ S. Viret, ${ }^{32}$ H. Xiao, ${ }^{32}$ Z. Tsamalaidze, ${ }^{33, i}$ C. Autermann, ${ }^{34}$ S. Beranek, ${ }^{34}$ M. Bontenackels, ${ }^{34}$ M. Edelhoff, ${ }^{34}$ L. Feld, ${ }^{34}$ A. Heister, ${ }^{34}$ O. Hindrichs, ${ }^{34}$ K. Klein, ${ }^{34}$ A. Ostapchuk, ${ }^{34}$ F. Raupach, ${ }^{34}$ J. Sammet, ${ }^{34}$ S. Schael,${ }^{34}$ H. Weber, ${ }^{34}$ B. Wittmer, ${ }^{34}$ V. Zhukov,,${ }^{34, f}$ M. Ata, ${ }^{35}$ M. Brodski, ${ }^{35}$ E. Dietz-Laursonn, ${ }^{35}$ D. Duchardt, ${ }^{35}$ M. Erdmann, ${ }^{35}$ R. Fischer, ${ }^{35}$ A. Güth, ${ }^{35}$ T. Hebbeker, ${ }^{35}$ C. Heidemann, ${ }^{35}$ K. Hoepfner, ${ }^{35}$ D. Klingebiel, ${ }^{35}$ S. Knutzen, ${ }^{35}$ P. Kreuzer, ${ }^{35}$ M. Merschmeyer, ${ }^{35}$ A. Meyer, ${ }^{35}$ P. Millet, ${ }^{35}$ M. Olschewski, ${ }^{35}$ K. Padeken,${ }^{35}$ P. Papacz, ${ }^{35}$ H. Reithler, ${ }^{35}$ S. A. Schmitz, ${ }^{35}$ L. Sonnenschein, ${ }^{35}$ D. Teyssier, ${ }^{35}$ S. Thüer, ${ }^{35}$ M. Weber ${ }^{35}$ V. Cherepanov, ${ }^{36}$ Y. Erdogan,${ }^{36}$ G. Flügge, ${ }^{36}$ H. Geenen ${ }^{36}$ M. Geisler, ${ }^{36}$ W. Haj Ahmad ${ }^{36}$ F. Hoehle, ${ }^{36}$ B. Kargoll, ${ }^{36}$ T. Kress, ${ }^{36}$ Y. Kuessel, ${ }^{36}$ A. Künsken, ${ }^{36}$ J. Lingemann, ${ }^{36, c}$ A. Nowack, ${ }^{36}$ I. M. Nugent, ${ }^{36}$ L. Perchalla, ${ }^{36}$ O. Pooth, ${ }^{36}$ A. Stahl,${ }^{36}$ I. Asin, ${ }^{37}$ N. Bartosik, ${ }^{37}$ J. Behr, ${ }^{37}$ U. Behrens, ${ }^{37}$ A. J. Bell,,${ }^{37}$ A. Bethani, ${ }^{37}$ K. Borras, ${ }^{37}$

A. Burgmeier, ${ }^{37}$ A. Cakir, ${ }^{37}$ L. Calligaris,${ }^{37}$ A. Campbell, ${ }^{37}$ S. Choudhury, ${ }^{37}$ F. Costanza, ${ }^{37}$ C. Diez Pardos, ${ }^{37}$ G. Dolinska,${ }^{37}$ S. Dooling, ${ }^{37}$ T. Dorland, ${ }^{37}$ G. Eckerlin, ${ }^{37}$ D. Eckstein, ${ }^{37}$ T. Eichhorn, ${ }^{37}$ G. Flucke, ${ }^{37}$ J. Garay Garcia, ${ }^{37}$ A. Geiser, ${ }^{37}$ P. Gunnellini, ${ }^{37}$ J. Hauk,${ }^{37}$ M. Hempel, ${ }^{37, p}$ H. Jung, ${ }^{37}$ A. Kalogeropoulos, ${ }^{37}$ M. Kasemann, ${ }^{37}$ P. Katsas, ${ }^{37}$ J. Kieseler, ${ }^{37}$ C. Kleinwort, ${ }^{37}$ I. Korol, ${ }^{37}$ D. Krücker, ${ }^{37}$ W. Lange, ${ }^{37}$ J. Leonard, ${ }^{37}$ K. Lipka, ${ }^{37}$ A. Lobanov, ${ }^{37}$ W. Lohmann, ${ }^{37, p}$ B. Lutz, ${ }^{37}$

R. Mankel, ${ }^{37}$ I. Marfin, ${ }^{37, p}$ I.-A. Melzer-Pellmann, ${ }^{37}$ A. B. Meyer, ${ }^{37}$ G. Mittag, ${ }^{37}$ J. Mnich, ${ }^{37}$ A. Mussgiller,${ }^{37}$ S. Naumann-Emme, ${ }^{37}$ A. Nayak, ${ }^{37}$ E. Ntomari, ${ }^{37}$ H. Perrey, ${ }^{37}$ D. Pitzl, ${ }^{37}$ R. Placakyte, ${ }^{37}$ A. Raspereza, ${ }^{37}$

P. M. Ribeiro Cipriano, ${ }^{37}$ B. Roland, ${ }^{37}$ E. Ron, ${ }^{37}$ M. Ö. Sahin, ${ }^{37}$ J. Salfeld-Nebgen, ${ }^{37}$ P. Saxena, ${ }^{37}$ T. Schoerner-Sadenius,${ }^{37}$ M. Schröder, ${ }^{37}$ C. Seitz, ${ }^{37}$ S. Spannagel, ${ }^{37}$ A. D. R. Vargas Trevino, ${ }^{37}$ R. Walsh, ${ }^{37}$ C. Wissing, ${ }^{37}$ M. Aldaya Martin, ${ }^{38}$

V. Blobel, ${ }^{38}$ M. Centis Vignali, ${ }^{38}$ A. R. Draeger, ${ }^{38}$ J. Erfle, ${ }^{38}$ E. Garutti, ${ }^{38}$ K. Goebel, ${ }^{38}$ M. Görner, ${ }^{38}$ J. Haller, ${ }^{38}$ M. Hoffmann, ${ }^{38}$ R. S. Höing, ${ }^{38}$ A. Junkes,${ }^{38}$ H. Kirschenmann, ${ }^{38}$ R. Klanner, ${ }^{38}$ R. Kogler, ${ }^{38}$ J. Lange, ${ }^{38}$ T. Lapsien, ${ }^{38}$ T. Lenz, ${ }^{38}$ I. Marchesini, ${ }^{38}$ J. Ott, ${ }^{38}$ T. Peiffer, ${ }^{38}$ A. Perieanu, ${ }^{38}$ N. Pietsch, ${ }^{38}$ J. Poehlsen, ${ }^{38}$ T. Poehlsen, ${ }^{38}$ D. Rathjens, ${ }^{38}$ C. Sander, ${ }^{38}$ H. Schettler, ${ }^{38}$ P. Schleper, ${ }^{38}$ E. Schlieckau, ${ }^{38}$ A. Schmidt, ${ }^{38}$ M. Seidel,${ }^{38}$ V. Sola, ${ }^{38}$ H. Stadie,${ }^{38}$ G. Steinbrück, ${ }^{38}$ D. Troendle, ${ }^{38}$ E. Usai, ${ }^{38}$ L. Vanelderen, ${ }^{38}$ A. Vanhoefer, ${ }^{38}$ C. Barth,${ }^{39}$ C. Baus, ${ }^{39}$ J. Berger, ${ }^{39}$ C. Böser, ${ }^{39}$ E. Butz, ${ }^{39}$ T. Chwalek, ${ }^{39}$ W. De Boer, ${ }^{39}$ A. Descroix, ${ }^{39}$ A. Dierlamm, ${ }^{39}$ M. Feindt, ${ }^{39}$ F. Frensch, ${ }^{39}$ M. Giffels, ${ }^{39}$ A. Gilbert, ${ }^{39}$ F. Hartmann, ${ }^{39, \mathrm{c}}$ T. Hauth, ${ }^{39, \mathrm{c}}$ U. Husemann, ${ }^{39}$ I. Katkov, ${ }^{39, \mathrm{f}}$ A. Kornmayer, ${ }^{39, \mathrm{c}}$ E. Kuznetsova, ${ }^{39}$ P. Lobelle Pardo, ${ }^{39}$ M. U. Mozer, ${ }^{39}$ T. Müller, ${ }^{39}$ Th. Müller, ${ }^{39}$ A. Nürnberg, ${ }^{39}$ G. Quast, ${ }^{39}$ K. Rabbertz, ${ }^{39}$ S. Röcker, ${ }^{39}$ H. J. Simonis, ${ }^{39}$ F. M. Stober, ${ }^{39}$ R. Ulrich, ${ }^{39}$ J. Wagner-Kuhr, ${ }^{39}$ S. Wayand,${ }^{39}$ T. Weiler ${ }^{39}$ R. Wolf, ${ }^{39}$ G. Anagnostou, ${ }^{40}$ G. Daskalakis, ${ }^{40}$ T. Geralis, ${ }^{40}$ V. A. Giakoumopoulou, ${ }^{40}$ A. Kyriakis, ${ }^{40}$ D. Loukas,${ }^{40}$ A. Markou, ${ }^{40}$ C. Markou, ${ }^{40}$ A. Psallidas, ${ }^{40}$ I. Topsis-Giotis, ${ }^{40}$ A. Agapitos, ${ }^{41}$ S. Kesisoglou, ${ }^{41}$ A. Panagiotou, ${ }^{41}$ N. Saoulidou, ${ }^{41}$ E. Stiliaris,${ }^{41}$ X. Aslanoglou, ${ }^{42}$ 
I. Evangelou, ${ }^{42}$ G. Flouris, ${ }^{42}$ C. Foudas, ${ }^{42}$ P. Kokkas, ${ }^{42}$ N. Manthos, ${ }^{42}$ I. Papadopoulos, ${ }^{42}$ E. Paradas,${ }^{42}$ J. Strologas, ${ }^{42}$ G. Bencze, ${ }^{43}$ C. Hajdu, ${ }^{43}$ P. Hidas, ${ }^{43}$ D. Horvath,${ }^{43, q}$ F. Sikler, ${ }^{43}$ V. Veszpremi, ${ }^{43}$ G. Vesztergombi, ${ }^{43, r}$ A. J. Zsigmond, ${ }^{43}$ N. Beni, ${ }^{44}$ S. Czellar, ${ }^{44}$ J. Karancsi, ${ }^{44, s}$ J. Molnar, ${ }^{44}$ J. Palinkas, ${ }^{44}$ Z. Szillasi, ${ }^{44}$ A. Makovec, ${ }^{45}$ P. Raics, ${ }^{45}$ Z. L. Trocsanyi, ${ }^{45}$ B. Ujvari, ${ }^{45}$ S. K. Swain, ${ }^{46}$ S. B. Beri,${ }^{47}$ V. Bhatnagar, ${ }^{47}$ R. Gupta,${ }^{47}$ U. Bhawandeep, ${ }^{47}$ A. K. Kalsi, ${ }^{47}$ M. Kaur, ${ }^{47}$ R. Kumar,${ }^{47}$ M. Mittal, ${ }^{47}$ N. Nishu, ${ }^{47}$ J. B. Singh, ${ }^{47}$ Ashok Kumar, ${ }^{48}$ Arun Kumar ${ }^{48}$ S. Ahuja ${ }^{48}$ A. Bhardwaj, ${ }^{48}$ B. C. Choudhary, ${ }^{48}$ A. Kumar, ${ }^{48}$ S. Malhotra, ${ }^{48}$ M. Naimuddin, ${ }^{48}$ K. Ranjan, ${ }^{48}$ V. Sharma, ${ }^{48}$ S. Banerjee, ${ }^{49}$ S. Bhattacharya, ${ }^{49}$ K. Chatterjee,${ }^{49}$ S. Dutta, ${ }^{49}$ B. Gomber, ${ }^{49}$ Sa. Jain, ${ }^{49}$ Sh. Jain, ${ }^{49}$ R. Khurana, ${ }^{49}$ A. Modak ${ }^{49}$ S. Mukherjee, ${ }^{49}$ D. Roy, ${ }^{49}$ S. Sarkar ${ }^{49}$ M. Sharan, ${ }^{49}$ A. Abdulsalam, ${ }^{50}$ D. Dutta, ${ }^{50}$ S. Kailas, ${ }^{50}$ V. Kumar, ${ }^{50}$ A. K. Mohanty, ${ }^{50, c}$ L. M. Pant, ${ }^{50}$ P. Shukla, ${ }^{50}$ A. Topkar, ${ }^{50}$ T. Aziz, ${ }^{51}$ S. Banerjee, ${ }^{51}$ S. Bhowmik,${ }^{51, t}$ R. M. Chatterjee, ${ }^{51}$ R. K. Dewanjee, ${ }^{51}$ S. Dugad, ${ }^{51}$ S. Ganguly, ${ }^{51}$ S. Ghosh ${ }^{51}$ M. Guchait, ${ }^{51}$ A. Gurtu, ${ }^{51, u}$ G. Kole, ${ }^{51}$ S. Kumar,${ }^{51}$ M. Maity, ${ }^{51, t}$ G. Majumder, ${ }^{51}$ K. Mazumdar, ${ }^{51}$ G. B. Mohanty, ${ }^{51}$ B. Parida, ${ }^{51}$ K. Sudhakar, ${ }^{51}$ N. Wickramage,${ }^{51, v}$ H. Bakhshiansohi, ${ }^{52}$ H. Behnamian, ${ }^{52}$ S. M. Etesami, ${ }^{52, w}$ A. Fahim ${ }^{52, x}$ R. Goldouzian, ${ }^{52}$ M. Khakzad, ${ }^{52}$ M. Mohammadi Najafabadi ${ }^{52}$ M. Naseri, ${ }^{52}$ S. Paktinat Mehdiabadi, ${ }^{52}$ F. Rezaei Hosseinabadi, ${ }^{52}$ B. Safarzadeh,${ }^{52, y}$ M. Zeinali, ${ }^{52}$ M. Felcini, ${ }^{53}$ M. Grunewald, ${ }^{53}$ M. Abbrescia, ${ }^{54 a, 54 b}$ C. Calabria, ${ }^{54 a, 54 b}$ S. S. Chhibra, ${ }^{54 a, 54 b}$ A. Colaleo, ${ }^{54 a}$ D. Creanza, ${ }^{54 a, 54 c}$ N. De Filippis, ${ }^{54 a, 54 c}$ M. De Palma, ${ }^{54 a, 54 b}$ L. Fiore, ${ }^{54 a}$ G. Iaselli, ${ }^{54 a, 54 c}$ G. Maggi, ${ }^{54 a, 54 c}$ M. Maggi, ${ }^{54 a}$ S. My, ${ }^{54,54 c}$ S. Nuzzo, ${ }^{54 a, 54 b}$ A. Pompili, ${ }^{54 a, 54 b}$ G. Pugliese, ${ }^{54 a, 54 c}$ R. Radogna,${ }^{54,54 b, c}$ G. Selvaggi, ${ }^{54,54 b}$ A. Sharma,${ }^{54 a}$ L. Silvestris, ${ }^{54 a, c}$ R. Venditti, ${ }^{54 a, 54 b}$ G. Abbiendi, ${ }^{55 a}$ A. C. Benvenuti, ${ }^{55 a}$

D. Bonacorsi ${ }^{55 a, 55 b}$ S. Braibant-Giacomelli, ${ }^{55 a, 55 b}$ L. Brigliadori, ${ }^{55 a, 55 b}$ R. Campanini, ${ }^{55 a, 55 b}$ P. Capiluppi, ${ }^{55 a, 55 b}$

A. Castro, ${ }^{55 a, 55 b}$ F. R. Cavallo, ${ }^{55 a}$ G. Codispoti, ${ }^{55 a, 55 b}$ M. Cuffiani, ${ }^{55 a, 55 b}$ G. M. Dallavalle, ${ }^{55 a}$ F. Fabbri, ${ }^{55 a}$ A. Fanfani,${ }^{55 a, 55 b}$ D. Fasanella, ${ }^{55 a, 55 b}$ P. Giacomelli, ${ }^{55 a}$ C. Grandi, ${ }^{55 a}$ L. Guiducci, ${ }^{55 a, 55 b}$ S. Marcellini, ${ }^{55 a}$ G. Masetti, ${ }^{55 a}$ A. Montanari, ${ }^{55 a}$

F. L. Navarria, ${ }^{55 a, 55 b}$ A. Perrotta, ${ }^{55 a}$ F. Primavera, ${ }^{55 a, 55 b}$ A. M. Rossi ${ }^{55 a, 55 b}$ T. Rovelli, ${ }^{55 a, 55 b}$ G. P. Siroli, ${ }^{55 a, 55 b}$ N. Tosi, ${ }^{55 a, 55 b}$ R. Travaglini, ${ }^{55 \mathrm{a}, 55 \mathrm{~b}}$ S. Albergo, ${ }^{56 a, 56 \mathrm{~b}}$ G. Cappello, ${ }^{56 \mathrm{a}}$ M. Chiorboli, ${ }^{56 a, 56 \mathrm{~b}}$ S. Costa, ${ }^{56 \mathrm{a}, 56 \mathrm{~b}}$ F. Giordano, ${ }^{56 \mathrm{a}, \mathrm{c}}$ R. Potenza, ${ }^{56 \mathrm{a}, 56 \mathrm{~b}}$ A. Tricomi, ${ }^{56 a, 56 b}$ C. Tuve, ${ }^{56,56 b}$ G. Barbagli, ${ }^{57 a}$ V. Ciulli, ${ }^{57 a, 57 b}$ C. Civinini,${ }^{57 a}$ R. D'Alessandro, ${ }^{57 a, 57 b}$ E. Focardi, ${ }^{57 a, 57 b}$ E. Gallo, ${ }^{57 a}$ S. Gonzi, ${ }^{57 a, 57 b}$ V. Gori, ${ }^{57 a, 57 b, c}$ P. Lenzi,${ }^{57 a, 57 b}$ M. Meschini, ${ }^{57 a}$ S. Paoletti, ${ }^{57 a}$ G. Sguazzoni, ${ }^{57 a}$ A. Tropiano, ${ }^{57 a, 57 b}$ L. Benussi, ${ }^{58}$ S. Bianco, ${ }^{58}$ F. Fabbri, ${ }^{58}$ D. Piccolo,${ }^{58}$ R. Ferretti, ${ }^{59 a, 59 b}$ F. Ferro,${ }^{59 a}$ M. Lo Vetere,${ }^{59 a, 59 b}$ E. Robutti, ${ }^{59 a}$ S. Tosi, ${ }^{59 a, 59 b}$ M. E. Dinardo, ${ }^{60 a, 60 b}$ S. Fiorendi, ${ }^{60 a, 60 b}$ S. Gennai, ${ }^{60 a, c}$ R. Gerosa,${ }^{60 a, 60 b, c}$ A. Ghezzi, ${ }^{60 a, 60 b}$ P. Govoni, ${ }^{60 a, 60 b}$ M. T. Lucchini, ${ }^{60 \mathrm{a}, 60 \mathrm{~b}, \mathrm{c}}$ S. Malvezzi, ${ }^{60 \mathrm{a}}$ R. A. Manzoni ${ }^{60 \mathrm{a}, 60 \mathrm{~b}}$ A. Martelli, ${ }^{60 \mathrm{a}, 60 \mathrm{~b}}$ B. Marzocchi, ${ }^{60 \mathrm{a}, 60 \mathrm{~b}, \mathrm{c}}$ D. Menasce, ${ }^{60 \mathrm{a}}$ L. Moroni, ${ }^{60 \mathrm{a}}$ M. Paganoni, ${ }^{60 \mathrm{a}, 60 \mathrm{~b}}$ D. Pedrini, ${ }^{60 \mathrm{a}}$ S. Ragazzi, ${ }^{60 \mathrm{a}, 60 \mathrm{~b}}$ N. Redaelli, ${ }^{60 \mathrm{a}}$ T. Tabarelli de Fatis ${ }^{60 \mathrm{a}, 60 \mathrm{~b}}$ S. Buontempo, ${ }^{61 \mathrm{a}}$ N. Cavallo, ${ }^{61 \mathrm{a}, 61 \mathrm{c}}$ S. Di Guida, ${ }^{61 a, 61 \mathrm{~d}, \mathrm{c}}$ F. Fabozzi ${ }^{61 \mathrm{a}, 61 \mathrm{c}}$ A. O. M. Iorio, ${ }^{61 \mathrm{a}, 61 \mathrm{~b}}$ L. Lista, ${ }^{61 \mathrm{a}}$ S. Meola,${ }^{61 \mathrm{a}, 61 \mathrm{~d}, \mathrm{c}}$ M. Merola, ${ }^{61 \mathrm{a}}$ P. Paolucci, ${ }^{61 a, c}$ P. Azzi, ${ }^{62 a}$ N. Bacchetta, ${ }^{62 a}$ M. Bellato, ${ }^{62 a}$ D. Bisello,${ }^{6 a, 62 b}$ R. Carlin, ${ }^{62 a, 62 b}$ P. Checchia, ${ }^{62 a}$

M. Dall'Osso, ${ }^{62 a, 62 b}$ T. Dorigo, ${ }^{62 a}$ M. Galanti, ${ }^{62 a, 62 b}$ F. Gasparini, ${ }^{62 a, 62 b}$ U. Gasparini, ${ }^{62 a, 62 b}$ P. Giubilato, ${ }^{62 a, 62 b}$

A. Gozzelino, ${ }^{62 \mathrm{a}}$ K. Kanishchev, ${ }^{62 \mathrm{a}, 62 \mathrm{c}}$ S. Lacaprara, ${ }^{62 \mathrm{a}}$ M. Margoni, ${ }^{62 \mathrm{a}, 62 \mathrm{~b}}$ A. T. Meneguzzo, ${ }^{62 \mathrm{a}, 62 \mathrm{~b}}$ J. Pazzini, ${ }^{62 \mathrm{a}, 62 \mathrm{~b}}$ M. Pegoraro, ${ }^{62 \mathrm{a}}$ N. Pozzobon, ${ }^{62 \mathrm{a}, 62 \mathrm{~b}}$ P. Ronchese, ${ }^{62 \mathrm{a}, 62 \mathrm{~b}}$ F. Simonetto, ${ }^{62 \mathrm{a}, 62 \mathrm{~b}}$ E. Torassa, ${ }^{62 \mathrm{a}}$ M. Tosi ${ }^{62 \mathrm{a}, 62 \mathrm{~b}}$ P. Zotto, ${ }^{62 \mathrm{a}, 62 \mathrm{~b}}$ A. Zucchetta, ${ }^{62 a, 62 b}$ G. Zumerle, ${ }^{62 a, 62 b}$ M. Gabusi, ${ }^{63 a, 63 b}$ S. P. Ratti, ${ }^{63 a, 63 b}$ V. Re ${ }^{63 a}$ C. Riccardi ${ }^{63 a, 63 b}$ P. Salvini, ${ }^{63 a}$ P. Vitulo, ${ }^{63 a, 63 b}$ M. Biasini, ${ }^{64 a, 64 b}$ G. M. Bilei, ${ }^{64 a}$ D. Ciangottini, ${ }^{64 a, 64 b, c}$ L. Fanò ${ }^{64 a, 64 b}$ P. Lariccia, ${ }^{64 a, 64 b}$ G. Mantovani, ${ }^{64 a, 64 b}$ M. Menichelli, ${ }^{64 a}$ A. Saha, ${ }^{64 a}$ A. Santocchia,${ }^{64 a, 64 b}$ A. Spiezia,${ }^{64,64 b, c}$ K. Androsov,${ }^{65 a, z}$ P. Azzurri, ${ }^{65 a}$ G. Bagliesi, ${ }^{65 a}$ J. Bernardini, ${ }^{65 a}$ T. Boccali, ${ }^{65 a}$ G. Broccolo,${ }^{65 a, 65 c}$ R. Castaldi, ${ }^{65 a}$ M. A. Ciocci, ${ }^{65 a, z}$ R. Dell'Orso, ${ }^{65 a}$ S. Donato, ${ }^{65 a, 65 c, c}$ F. Fiori, ${ }^{65 a, 65 c}$ L. Foà, ${ }^{65 a, 65 c}$ A. Giassi, ${ }^{65 a}$ M. T. Grippo,${ }^{65 a, z}$ F. Ligabue, ${ }^{65 a, 65 c}$ T. Lomtadze,${ }^{65 a}$ L. Martini, ${ }^{65 a, 65 b}$ A. Messineo, ${ }^{65 a, 65 b}$ C. S. Moon,${ }^{65 a, a a}$ F. Palla, ${ }^{65 a, c}$ A. Rizzi ${ }^{65 a, 65 b}$ A. Savoy-Navarro, ${ }^{65 a, b b}$ A. T. Serban, ${ }^{65 a}$ P. Spagnolo, ${ }^{65 a}$ P. Squillacioti ${ }^{65 a, z}$ R. Tenchini, ${ }^{65 a}$ G. Tonelli, ${ }^{65 a, 65 b}$ A. Venturi, ${ }^{65 a}$ P. G. Verdini ${ }^{65 a}$ C. Vernieri ${ }^{65 a, 65 c, c}$ L. Barone, ${ }^{66 a, 66 b}$ F. Cavallari, ${ }^{66 \mathrm{a}}$ G. D’imperio, ${ }^{66 a, 66 \mathrm{~b}}$ D. Del Re ${ }^{66 \mathrm{a}, 66 \mathrm{~b}} \mathrm{M}$. Diemoz, ${ }^{66 \mathrm{a}}$ C. Jorda ${ }^{66 \mathrm{a}}$ E. Longo, ${ }^{66 \mathrm{a}, 66 \mathrm{~b}}$ F. Margaroli, ${ }^{66 \mathrm{a}, 66 \mathrm{~b}}$ P. Meridiani, ${ }^{66 a}$ F. Micheli, ${ }^{66 a, 66 b, c}$ S. Nourbakhsh, ${ }^{66 a, 66 b}$ G. Organtini, ${ }^{66 a, 66 b}$ R. Paramatti, ${ }^{66 a}$ S. Rahatlou, ${ }^{66 a, 66 b}$ C. Rovelli, ${ }^{66 a}$ F. Santanastasio, ${ }^{66 a, 66 b}$ L. Soffi, ${ }^{66 a, 66 b, c}$ P. Traczyk,${ }^{66 a, 66 b, c}$ N. Amapane, ${ }^{67 a, 67 b}$ R. Arcidiacono, ${ }^{67 a, 67 c}$ S. Argiro, ${ }^{67 a, 67 b}$ M. Arneodo, ${ }^{67,67 \mathrm{c}}$ R. Bellan, ${ }^{67 \mathrm{a}, 67 \mathrm{~b}}$ C. Biino, ${ }^{67 \mathrm{a}}$ N. Cartiglia, ${ }^{67 \mathrm{a}}$ S. Casasso,${ }^{67 a, 67 b, c}$ M. Costa, ${ }^{67 \mathrm{a}, 67 \mathrm{~b}}$ A. Degano, ${ }^{67 \mathrm{a}, 67 \mathrm{~b}}$ N. Demaria, ${ }^{67 a}$ L. Finco, ${ }^{67 a, 67 b, c}$ C. Mariotti, ${ }^{67 a}$ S. Maselli, ${ }^{67 a}$ E. Migliore, ${ }^{67 a, 67 b}$ V. Monaco, ${ }^{67 a, 67 b}$ M. Musich, ${ }^{67 a}$ M. M. Obertino, ${ }^{67 a, 67 c, c}$ G. Ortona ${ }^{67 a, 67 b}$ L. Pacher, ${ }^{67 a, 67 b}$ N. Pastrone,${ }^{67 a}$ M. Pelliccioni, ${ }^{67 a}$ G. L. Pinna Angioni, ${ }^{67 a, 67 b}$ A. Potenza,${ }^{67 a, 67 b}$ A. Romero, ${ }^{67 a, 67 b}$ M. Ruspa, ${ }^{67 a, 67 c}$ R. Sacchi, ${ }^{67 a, 67 b}$ A. Solano,${ }^{67 a, 67 b}$ A. Staiano, ${ }^{67 a}$ U. Tamponi, ${ }^{67 a}$ S. Belforte, ${ }^{68 a}$ V. Candelise,${ }^{68 a, 68 b, c}$ M. Casarsa, ${ }^{68 a}$ F. Cossutti, ${ }^{68 a}$ G. Della Ricca,${ }^{68 a, 68 b}$ B. Gobbo, ${ }^{68 a}$ C. La Licata, ${ }^{68 a, 68 b}$ M. Marone ${ }^{68 a, 68 b}$ A. Schizzi, ${ }^{68 a, 68 b}$ T. Umer, ${ }^{68 a, 68 b}$ A. Zanetti, ${ }^{68 a}$ S. Chang, ${ }^{69}$ A. Kropivnitskaya, ${ }^{69}$ S. K. Nam, ${ }^{69}$ D. H. Kim, ${ }^{70}$ G. N. Kim, ${ }^{70}$ M. S. Kim, ${ }^{70}$ D. J. Kong, ${ }^{70}$ S. Lee, ${ }^{70}$ Y. D. Oh ${ }^{70}$ H. Park, ${ }^{70}$ A. Sakharov, ${ }^{70}$ D. C. Son, ${ }^{70}$ T. J. Kim, ${ }^{71}$ J. Y. Kim, ${ }^{72}$ 
S. Song, ${ }^{72}$ S. Choi,${ }^{73}$ D. Gyun, ${ }^{73}$ B. Hong, ${ }^{73}$ M. Jo, ${ }^{73}$ H. Kim, ${ }^{73}$ Y. Kim, ${ }^{73}$ B. Lee, ${ }^{73}$ K. S. Lee, ${ }^{73}$ S. K. Park, ${ }^{73}$ Y. Roh,${ }^{73}$ M. Choi,${ }^{74}$ J. H. Kim ${ }^{74}$ I. C. Park,${ }^{74}$ G. Ryu, ${ }^{74}$ M. S. Ryu,${ }^{74}$ Y. Choi, ${ }^{75}$ Y. K. Choi, ${ }^{75}$ J. Goh ${ }^{75}$ D. Kim,${ }^{75}$ E. Kwon, ${ }^{75}$ J. Lee, ${ }^{75}$ H. Seo, ${ }^{75}$ I. Yu, ${ }^{75}$ A. Juodagalvis, ${ }^{76}$ J. R. Komaragiri, ${ }^{77}$ M. A. B. Md Ali, ${ }^{77}$ E. Casimiro Linares, ${ }^{78}$ H. Castilla-Valdez, ${ }^{78}$ E. De La Cruz-Burelo, ${ }^{78}$ I. Heredia-de La Cruz, ${ }^{78, c c}$ A. Hernandez-Almada ${ }^{78}$ R. Lopez-Fernandez, ${ }^{78}$

A. Sanchez-Hernandez, ${ }^{78}$ S. Carrillo Moreno, ${ }^{79}$ F. Vazquez Valencia, ${ }^{79}$ I. Pedraza, ${ }^{80}$ H. A. Salazar Ibarguen, ${ }^{80}$ A. Morelos Pineda, ${ }^{81}$ D. Krofcheck, ${ }^{82}$ P. H. Butler ${ }^{83}$ S. Reucroft, ${ }^{83}$ A. Ahmad, ${ }^{84}$ M. Ahmad ${ }^{84}$ Q. Hassan,${ }^{84}$ H. R. Hoorani,${ }^{84}$ W. A. Khan, ${ }^{84}$ T. Khurshid, ${ }^{84}$ M. Shoaib,${ }^{84}$ H. Bialkowska, ${ }^{85}$ M. Bluj ${ }^{85}$ B. Boimska, ${ }^{85}$ T. Frueboes, ${ }^{85}$ M. Górski ${ }^{85}$ M. Kazana, ${ }^{85}$ K. Nawrocki, ${ }^{85}$ K. Romanowska-Rybinska, ${ }^{85}$ M. Szleper, ${ }^{85}$ P. Zalewski, ${ }^{85}$ G. Brona, ${ }^{86}$ K. Bunkowski, ${ }^{86}$ M. Cwiok ${ }^{86}$ W. Dominik, ${ }^{86}$ K. Doroba,${ }^{86}$ A. Kalinowski, ${ }^{86}$ M. Konecki, ${ }^{86}$ J. Krolikowski, ${ }^{86}$ M. Misiura, ${ }^{86}$ M. Olszewski, ${ }^{86}$ W. Wolszczak, ${ }^{86}$ P. Bargassa, ${ }^{87}$ C. Beirão Da Cruz E Silva, ${ }^{87}$ P. Faccioli, ${ }^{87}$ P. G. Ferreira Parracho, ${ }^{87}$ M. Gallinaro, ${ }^{87}$ L. Lloret Iglesias, ${ }^{87}$ F. Nguyen, ${ }^{87}$ J. Rodrigues Antunes, ${ }^{87}$ J. Seixas, ${ }^{87}$ J. Varela,${ }^{87}$ P. Vischia, ${ }^{87}$ S. Afanasiev,${ }^{88}$ P. Bunin,${ }^{88}$ M. Gavrilenko, ${ }^{88}$ I. Golutvin, ${ }^{88}$ I. Gorbunov, ${ }^{88}$ A. Kamenev, ${ }^{88}$ V. Karjavin, ${ }^{88}$ V. Konoplyanikov, ${ }^{88}$ A. Lanev, ${ }^{88}$ A. Malakhov, ${ }^{88}$ V. Matveev, ${ }^{88, d d}$ P. Moisenz, ${ }^{88}$ V. Palichik, ${ }^{88}$ V. Perelygin, ${ }^{88}$ S. Shmatov, ${ }^{88}$ N. Skatchkov, ${ }^{88}$ V. Smirnov, ${ }^{88}$ A. Zarubin, ${ }^{88}$ V. Golovtsov, ${ }^{89}$ Y. Ivanov, ${ }^{89}$ V. Kim,,${ }^{89}$ ee P. Levchenko, ${ }^{89}$ V. Murzin, ${ }^{89}$ V. Oreshkin, ${ }^{89}$ I. Smirnov, ${ }^{89}$ V. Sulimov, ${ }^{89}$ L. Uvarov, ${ }^{89}$ S. Vavilov, ${ }^{89}$ A. Vorobyev, ${ }^{89}$ An. Vorobyev, ${ }^{89}$ Yu. Andreev, ${ }^{90}$ A. Dermenev, ${ }^{90}$ S. Gninenko, ${ }^{90}$ N. Golubev, ${ }^{90}$ M. Kirsanov, ${ }^{90}$ N. Krasnikov, ${ }^{90}$ A. Pashenkov, ${ }^{90}$ D. Tlisov, ${ }^{90}$ A. Toropin, ${ }^{90}$ V. Epshteyn, ${ }^{91}$ V. Gavrilov, ${ }^{91}$ N. Lychkovskaya, ${ }^{91}$ V. Popov, ${ }^{91}$ I. Pozdnyakov, ${ }^{91}$ G. Safronov,${ }^{91}$ S. Semenov, ${ }^{91}$ A. Spiridonov,${ }^{91}$ V. Stolin,${ }^{91}$ E. Vlasov, ${ }^{91}$ A. Zhokin, ${ }^{91}$ V. Andreev, ${ }^{92}$ M. Azarkin, ${ }^{92}$ I. Dremin, ${ }^{92}$ M. Kirakosyan, ${ }^{92}$ A. Leonidov, ${ }^{92}$ G. Mesyats, ${ }^{92}$ S. V. Rusakov, ${ }^{92}$ A. Vinogradov, ${ }^{92}$ A. Belyaev, ${ }^{93}$ E. Boos, ${ }^{93}$ V. Bunichev, ${ }^{93}$ M. Dubinin,,${ }^{93, f f}$ L. Dudko, ${ }^{93}$ A. Ershov, ${ }^{93}$ A. Gribushin, ${ }^{93}$ V. Klyukhin, ${ }^{93}$ O. Kodolova, ${ }^{93}$ I. Lokhtin, ${ }^{93}$ S. Obraztsov, ${ }^{93}$ V. Savrin, ${ }^{93}$ A. Snigirev, ${ }^{93}$ I. Azhgirey,${ }^{94}$ I. Bayshev, ${ }^{94}$ S. Bitioukov, ${ }^{94}$ V. Kachanov, ${ }^{94}$ A. Kalinin, ${ }^{94}$ D. Konstantinov, ${ }^{94}$ V. Krychkine, ${ }^{94}$ V. Petrov, ${ }^{94}$ R. Ryutin, ${ }^{94}$ A. Sobol, ${ }^{94}$ L. Tourtchanovitch, ${ }^{94}$ S. Troshin, ${ }^{94}$ N. Tyurin, ${ }^{94}$ A. Uzunian, ${ }^{94}$ A. Volkov, ${ }^{94}$ P. Adzic, ${ }^{95, g g}$ M. Ekmedzic, ${ }^{95}$ J. Milosevic, ${ }^{95}$ V. Rekovic, ${ }^{95}$ J. Alcaraz Maestre, ${ }^{96}$ C. Battilana, ${ }^{96}$ E. Calvo,${ }^{96}$ M. Cerrada, ${ }^{96}$ M. Chamizo Llatas, ${ }^{96}$ N. Colino, ${ }^{96}$ B. De La Cruz, ${ }^{96}$ A. Delgado Peris, ${ }^{96}$ D. Domínguez Vázquez, ${ }^{96}$ A. Escalante Del Valle, ${ }^{96}$ C. Fernandez Bedoya, ${ }^{96}$ J.P. Fernández Ramos, ${ }^{96}$ J. Flix, ${ }^{96}$ M. C. Fouz,${ }^{96}$ P. Garcia-Abia, ${ }^{96}$ O. Gonzalez Lopez, ${ }^{96}$ S. Goy Lopez, ${ }^{96}$

J. M. Hernandez, ${ }^{96}$ M. I. Josa, ${ }^{96}$ E. Navarro De Martino, ${ }^{96}$ A. Pérez-Calero Yzquierdo, ${ }^{96}$ J. Puerta Pelayo, ${ }^{96}$

A. Quintario Olmeda, ${ }^{96}$ I. Redondo, ${ }^{96}$ L. Romero, ${ }^{96}$ M. S. Soares, ${ }^{96}$ C. Albajar,${ }^{97}$ J. F. de Trocóniz, ${ }^{97}$ M. Missiroli, ${ }^{97}$

D. Moran, ${ }^{97}$ H. Brun, ${ }^{98}$ J. Cuevas, ${ }^{98}$ J. Fernandez Menendez, ${ }^{98}$ S. Folgueras, ${ }^{98}$ I. Gonzalez Caballero, ${ }^{98}$

J. A. Brochero Cifuentes, ${ }^{99}$ I. J. Cabrillo, ${ }^{99}$ A. Calderon, ${ }^{99}$ J. Duarte Campderros, ${ }^{99}$ M. Fernandez,${ }^{99}$ G. Gomez, ${ }^{99}$ A. Graziano, ${ }^{99}$ A. Lopez Virto, ${ }^{99}$ J. Marco, ${ }^{99}$ R. Marco, ${ }^{99}$ C. Martinez Rivero, ${ }^{99}$ F. Matorras, ${ }^{99}$ F. J. Munoz Sanchez, ${ }^{99}$ J. Piedra Gomez, ${ }^{99}$ T. Rodrigo, ${ }^{99}$ A. Y. Rodríguez-Marrero, ${ }^{99}$ A. Ruiz-Jimeno, ${ }^{99}$ L. Scodellaro, ${ }^{99}$ I. Vila, ${ }^{99}$

R. Vilar Cortabitarte, ${ }^{99}$ D. Abbaneo, ${ }^{100}$ E. Auffray, ${ }^{100}$ G. Auzinger,${ }^{100}$ M. Bachtis,${ }^{100}$ P. Baillon, ${ }^{100}$ A. H. Ball, ${ }^{100}$ D. Barney, ${ }^{100}$ A. Benaglia, ${ }^{100}$ J. Bendavid, ${ }^{100}$ L. Benhabib, ${ }^{100}$ J. F. Benitez, ${ }^{100}$ C. Bernet, ${ }^{100, h}$ P. Bloch,${ }^{100}$ A. Bocci, ${ }^{100}$ A. Bonato, ${ }^{100}$ O. Bondu, ${ }^{100}$ C. Botta, ${ }^{100}$ H. Breuker, ${ }^{100}$ T. Camporesi, ${ }^{100}$ G. Cerminara, ${ }^{100}$ S. Colafranceschi, ${ }^{100, \text { hh }}$ M. D'Alfonso, ${ }^{100}$ D. d'Enterria, ${ }^{100}$ A. Dabrowski, ${ }^{100}$ A. David,${ }^{100}$ F. De Guio, ${ }^{100}$ A. De Roeck, ${ }^{100}$ S. De Visscher, ${ }^{100}$ E. Di Marco, ${ }^{100}$ M. Dobson, ${ }^{100}$ M. Dordevic, ${ }^{100}$ N. Dupont-Sagorin, ${ }^{100}$ A. Elliott-Peisert, ${ }^{100}$ J. Eugster, ${ }^{100}$ G. Franzoni, ${ }^{100}$ W. Funk, ${ }^{100}$ D. Gigi, ${ }^{100}$ K. Gill, ${ }^{100}$ D. Giordano, ${ }^{100}$ M. Girone,${ }^{100}$ F. Glege,${ }^{100}$ R. Guida, ${ }^{100}$ S. Gundacker, ${ }^{100}$ M. Guthoff, ${ }^{100}$ J. Hammer, ${ }^{100}$ M. Hansen, ${ }^{100}$ P. Harris, ${ }^{100}$ J. Hegeman, ${ }^{100}$ V. Innocente, ${ }^{100}$ P. Janot, ${ }^{100}$ K. Kousouris ${ }^{100}$ K. Krajczar,${ }^{100}$ P. Lecoq,${ }^{100}$ C. Lourenço, ${ }^{100}$ N. Magini, ${ }^{100}$ L. Malgeri, ${ }^{100}$ M. Mannelli, ${ }^{100}$ J. Marrouche, ${ }^{100}$ L. Masetti,${ }^{100}$ F. Meijers,${ }^{100}$ S. Mersi, ${ }^{100}$ E. Meschi, ${ }^{100}$ F. Moortgat, ${ }^{100}$ S. Morovic, ${ }^{100}$ M. Mulders, ${ }^{100}$ P. Musella, ${ }^{100}$ L. Orsini, ${ }^{100}$ L. Pape,${ }^{100}$ E. Perez, ${ }^{100}$ L. Perrozzi, ${ }^{100}$ A. Petrilli, ${ }^{100}$ G. Petrucciani, ${ }^{100}$ A. Pfeiffer, ${ }^{100}$ M. Pierini, ${ }^{100}$ M. Pimiä, ${ }^{100}$ D. Piparo, ${ }^{100}$ M. Plagge,${ }^{100}$ A. Racz, ${ }^{100}$ G. Rolandi, ${ }^{100, \text { ii }}$ M. Rovere,${ }^{100}$ H. Sakulin, ${ }^{100}$ C. Schäfer, ${ }^{100}$ C. Schwick, ${ }^{100}$ A. Sharma, ${ }^{100}$ P. Siegrist ${ }^{100}$ P. Silva, ${ }^{100}$ M. Simon, ${ }^{100}$ P. Sphicas, ${ }^{100, j j}$ D. Spiga, ${ }^{100}$ J. Steggemann, ${ }^{100}$ B. Stieger, ${ }^{100}$ M. Stoye, ${ }^{100}$ Y. Takahashi, ${ }^{100}$ D. Treille, ${ }^{100}$ A. Tsirou, ${ }^{100}$ G. I. Veres, ${ }^{100, r}$ N. Wardle,${ }^{100}$ H. K. Wöhri, ${ }^{100}$ H. Wollny, ${ }^{100}$ W. D. Zeuner, ${ }^{100}$ W. Bertl, ${ }^{101}$ K. Deiters, ${ }^{101}$ W. Erdmann, ${ }^{101}$ R. Horisberger, ${ }^{101}$ Q. Ingram, ${ }^{101}$ H. C. Kaestli, ${ }^{101}$ D. Kotlinski, ${ }^{101}$ U. Langenegger,${ }^{101}$ D. Renker, ${ }^{101}$ T. Rohe, ${ }^{101}$ F. Bachmair, ${ }^{102}$ L. Bäni, ${ }^{102}$ L. Bianchini, ${ }^{102}$ M. A. Buchmann, ${ }^{102}$ B. Casal, ${ }^{102}$ N. Chanon, ${ }^{102}$ G. Dissertori, ${ }^{102}$ M. Dittmar, ${ }^{102}$ M. Donegà ${ }^{102}$ M. Dünser, ${ }^{102}$ P. Eller, ${ }^{102}$ C. Grab, ${ }^{102}$ D. Hits, ${ }^{102}$ J. Hoss, ${ }^{102}$

W. Lustermann, ${ }^{102}$ B. Mangano, ${ }^{102}$ A. C. Marini, ${ }^{102}$ P. Martinez Ruiz del Arbol, ${ }^{102}$ M. Masciovecchio, ${ }^{102}$ D. Meister, ${ }^{102}$ N. Mohr, ${ }^{102}$ C. Nägeli, ${ }^{102, k k}$ F. Nessi-Tedaldi, ${ }^{102}$ F. Pandolfi, ${ }^{102}$ F. Pauss, ${ }^{102}$ M. Peruzzi, ${ }^{102}$ M. Quittnat,${ }^{102}$ L. Rebane, ${ }^{102}$ 
M. Rossini, ${ }^{102}$ A. Starodumov, ${ }^{102,11}$ M. Takahashi, ${ }^{102}$ K. Theofilatos, ${ }^{102}$ R. Wallny, ${ }^{102}$ H. A. Weber, ${ }^{102}$ C. Amsler, ${ }^{103, m m}$ M. F. Canelli, ${ }^{103}$ V. Chiochia, ${ }^{103}$ A. De Cosa, ${ }^{103}$ A. Hinzmann, ${ }^{103}$ T. Hreus, ${ }^{103}$ B. Kilminster,${ }^{103}$ C. Lange, ${ }^{103}$ B. Millan Mejias, ${ }^{103}$ J. Ngadiuba, ${ }^{103}$ D. Pinna, ${ }^{103}$ P. Robmann, ${ }^{103}$ F. J. Ronga, ${ }^{103}$ S. Taroni, ${ }^{103}$ M. Verzetti, ${ }^{103}$ Y. Yang, ${ }^{103}$ M. Cardaci, ${ }^{104}$ K. H. Chen, ${ }^{104}$ C. Ferro, ${ }^{104}$ C. M. Kuo, ${ }^{104}$ W. Lin, ${ }^{104}$ Y. J. Lu, ${ }^{104}$ R. Volpe, ${ }^{104}$ S. S. Yu, ${ }^{104}$ P. Chang, ${ }^{105}$ Y. H. Chang, ${ }^{105}$ Y. W. Chang, ${ }^{105}$ Y. Chao, ${ }^{105}$ K. F. Chen, ${ }^{105}$ P. H. Chen, ${ }^{105}$ C. Dietz, ${ }^{105}$ U. Grundler, ${ }^{105}$ W.-S. Hou, ${ }^{105}$ K. Y. Kao, ${ }^{105}$ Y. F. Liu, ${ }^{105}$ R.-S. Lu, ${ }^{105}$ D. Majumder,${ }^{105}$ E. Petrakou, ${ }^{105}$ Y. M. Tzeng, ${ }^{105}$ R. Wilken, ${ }^{105}$ B. Asavapibhop, ${ }^{106}$ G. Singh, ${ }^{106}$ N. Srimanobhas, ${ }^{106}$ N. Suwonjandee, ${ }^{106}$ A. Adiguzel,${ }^{107}$ M. N. Bakirci, ${ }^{107, n n}$ S. Cerci, ${ }^{107, \text { oo }}$ C. Dozen, ${ }^{107}$ I. Dumanoglu, ${ }^{107}$ E. Eskut, ${ }^{107}$ S. Girgis, ${ }^{107}$ G. Gokbulut, ${ }^{107}$ E. Gurpinar, ${ }^{107}$ I. Hos, ${ }^{107}$ E. E. Kangal,,${ }^{107}$ A. Kayis Topaksu, ${ }^{107}$ G. Onengut, ${ }^{107, p p}$ K. Ozdemir, ${ }^{107}$ S. Ozturk, ${ }^{107, n n}$ A. Polatoz, ${ }^{107}$ D. Sunar Cerci, ${ }^{107, \text { oo }}$ B. Tali, ${ }^{107,00}$ H. Topakli, ${ }^{107, \text {,n }}$ M. Vergili, ${ }^{107}$ I. V. Akin, ${ }^{108}$ B. Bilin, ${ }^{108}$ S. Bilmis, ${ }^{108}$ H. Gamsizkan, ${ }^{108, q q}$ B. Isildak, ${ }^{108, \text { rr }}$ G. Karapinar, ${ }^{108, s s}$ K. Ocalan, ${ }^{108, t t}$ S. Sekmen, ${ }^{108}$ U. E. Surat, ${ }^{108}$ M. Yalvac, ${ }^{108}$ M. Zeyrek, ${ }^{108}$ E. A. Albayrak, ${ }^{109, \text { uu }}$ E. Gülmez, ${ }^{109}$ M. Kaya, ${ }^{109, v v}$ O. Kaya, ${ }^{109, \text { ww }}$ T. Yetkin, ${ }^{109, x x}$ K. Cankocak, ${ }^{110}$ F. I. Vardarl, ${ }^{110}$ L. Levchuk, ${ }^{111}$ P. Sorokin, ${ }^{111}$ J. J. Brooke, ${ }^{112}$ E. Clement, ${ }^{112}$ D. Cussans, ${ }^{112}$ H. Flacher, ${ }^{112}$ J. Goldstein, ${ }^{112}$ M. Grimes, ${ }^{112}$ G. P. Heath, ${ }^{112}$ H. F. Heath, ${ }^{112}$ J. Jacob, ${ }^{112}$ L. Kreczko, ${ }^{112}$ C. Lucas, ${ }^{112}$ Z. Meng, ${ }^{112}$ D. M. Newbold, ${ }^{112, y y}$ S. Paramesvaran, ${ }^{112}$ A. Poll, ${ }^{112}$ T. Sakuma, ${ }^{112}$ S. Senkin, ${ }^{112}$ V. J. Smith, ${ }^{112}$ T. Williams, ${ }^{112}$ K. W. Bell, ${ }^{113}$ A. Belyaev, ${ }^{113, z z}$ C. Brew, ${ }^{113}$ R. M. Brown, ${ }^{113}$ D. J. A. Cockerill, ${ }^{113}$ J. A. Coughlan, ${ }^{113}$ K. Harder, ${ }^{113}$ S. Harper, ${ }^{113}$ E. Olaiya, ${ }^{113}$ D. Petyt, ${ }^{113}$ C. H. Shepherd-Themistocleous, ${ }^{113}$ A. Thea,${ }^{113}$ I. R. Tomalin, ${ }^{113}$ W. J. Womersley, ${ }^{113}$ S. D. Worm, ${ }^{113}$ M. Baber,${ }^{114}$ R. Bainbridge,${ }^{114}$ O. Buchmuller, ${ }^{114}$ D. Burton, ${ }^{114}$ D. Colling, ${ }^{114}$ N. Cripps,${ }^{114}$ P. Dauncey, ${ }^{114}$ G. Davies, ${ }^{114}$ M. Della Negra, ${ }^{114}$ P. Dunne, ${ }^{114}$ W. Ferguson, ${ }^{114}$ J. Fulcher, ${ }^{114}$ D. Futyan, ${ }^{114}$ G. Hall, ${ }^{114}$ G. Iles,${ }^{114}$ M. Jarvis, ${ }^{114}$ G. Karapostoli, ${ }^{114}$ M. Kenzie, ${ }^{114}$ R. Lane, ${ }^{114}$ R. Lucas, ${ }^{114, y y}$ L. Lyons, ${ }^{114}$ A.-M. Magnan, ${ }^{114}$ S. Malik, ${ }^{114}$ B. Mathias, ${ }^{114}$ J. Nash, ${ }^{114}$ A. Nikitenko, ${ }^{14,11}$ J. Pela, ${ }^{114}$ M. Pesaresi, ${ }^{114}$ K. Petridis, ${ }^{114}$ D. M. Raymond, ${ }^{114}$ S. Rogerson, ${ }^{114}$ A. Rose, ${ }^{114}$ C. Seez, ${ }^{114}$ P. Sharp ${ }^{114, a}$ A. Tapper, ${ }^{114}$ M. Vazquez Acosta, ${ }^{114}$ T. Virdee, ${ }^{114}$ S. C. Zenz, ${ }^{114}$ J. E. Cole, ${ }^{115}$ P. R. Hobson, ${ }^{115}$ A. Khan, ${ }^{115}$ P. Kyberd, ${ }^{115}$ D. Leggat,${ }^{115}$ D. Leslie, ${ }^{115}$ I. D. Reid, ${ }^{115}$ P. Symonds, ${ }^{115}$ L. Teodorescu, ${ }^{115}$ M. Turner, ${ }^{115}$ J. Dittmann, ${ }^{116}$ K. Hatakeyama, ${ }^{116}$ A. Kasmi,${ }^{116}$ H. Liu, ${ }^{116}$ T. Scarborough, ${ }^{116}$ O. Charaf,,${ }^{117}$ S. I. Cooper,${ }^{117}$ C. Henderson, ${ }^{117}$ P. Rumerio, ${ }^{117}$ A. Avetisyan, ${ }^{118}$ T. Bose, ${ }^{118}$ C. Fantasia, ${ }^{118}$ P. Lawson, ${ }^{118}$ C. Richardson, ${ }^{118}$ J. Rohlf, ${ }^{118}$ J. St. John, ${ }^{118}$ L. Sulak, ${ }^{118}$ J. Alimena, ${ }^{119}$ E. Berry,${ }^{119}$ S. Bhattacharya, ${ }^{119}$ G. Christopher, ${ }^{119}$ D. Cutts, ${ }^{119}$ Z. Demiragli, ${ }^{119}$ N. Dhingra, ${ }^{119}$ A. Ferapontov, ${ }^{119}$ A. Garabedian, ${ }^{119}$ U. Heintz, ${ }^{119}$ G. Kukartsev, ${ }^{119}$ E. Laird, ${ }^{119}$ G. Landsberg, ${ }^{119}$ M. Luk, ${ }^{119}$ M. Narain, ${ }^{119}$ M. Segala, ${ }^{119}$ T. Sinthuprasith, ${ }^{119}$ T. Speer, ${ }^{119}$ J. Swanson, ${ }^{119}$ R. Breedon, ${ }^{120}$ G. Breto, ${ }^{120}$

M. Calderon De La Barca Sanchez, ${ }^{120}$ S. Chauhan, ${ }^{120}$ M. Chertok, ${ }^{120}$ J. Conway, ${ }^{120}$ R. Conway, ${ }^{120}$ P. T. Cox, ${ }^{120}$ R. Erbacher, ${ }^{120}$ M. Gardner, ${ }^{120}$ W. Ko, ${ }^{120}$ R. Lander, ${ }^{120}$ T. Miceli, ${ }^{120}$ M. Mulhearn, ${ }^{120}$ D. Pellett, ${ }^{120}$ J. Pilot, ${ }^{120}$ F. Ricci-Tam, ${ }^{120}$ M. Searle, ${ }^{120}$ S. Shalhout, ${ }^{120}$ J. Smith, ${ }^{120}$ M. Squires, ${ }^{120}$ D. Stolp, ${ }^{120}$ M. Tripathi, ${ }^{120}$ S. Wilbur, ${ }^{120}$ R. Yohay, ${ }^{120}$ R. Cousins, ${ }^{121}$ P. Everaerts, ${ }^{121}$ C. Farrell, ${ }^{121}$ J. Hauser, ${ }^{121}$ M. Ignatenko, ${ }^{121}$ G. Rakness, ${ }^{121}$ E. Takasugi, ${ }^{121}$ V. Valuev, ${ }^{121}$ M. Weber, ${ }^{121}$ K. Burt, ${ }^{122}$ R. Clare, ${ }^{122}$ J. Ellison, ${ }^{122}$ J. W. Gary, ${ }^{122}$ G. Hanson, ${ }^{122}$ J. Heilman, ${ }^{122}$ M. Ivova Rikova, ${ }^{122}$ P. Jandir, ${ }^{122}$ E. Kennedy, ${ }^{122}$ F. Lacroix, ${ }^{122}$ O. R. Long, ${ }^{122}$ A. Luthra, ${ }^{122}$ M. Malberti, ${ }^{122}$ M. Olmedo Negrete, ${ }^{122}$ A. Shrinivas, ${ }^{122}$ S. Sumowidagdo, ${ }^{122}$ S. Wimpenny, ${ }^{122}$ J. G. Branson, ${ }^{123}$ G. B. Cerati, ${ }^{123}$ S. Cittolin, ${ }^{123}$ R. T. D’Agnolo, ${ }^{123}$ A. Holzner, ${ }^{123}$ R. Kelley, ${ }^{123}$ D. Klein, ${ }^{123}$ D. Kovalskyi, ${ }^{123}$ J. Letts, ${ }^{123}$ I. Macneill, ${ }^{123}$ D. Olivito, ${ }^{123}$ S. Padhi, ${ }^{123}$ C. Palmer, ${ }^{123}$ M. Pieri, ${ }^{123}$ M. Sani, ${ }^{123}$ V. Sharma, ${ }^{123}$ S. Simon, ${ }^{123}$ E. Sudano, ${ }^{123}$ Y. Tu, ${ }^{123}$ A. Vartak,${ }^{123}$ C. Welke, ${ }^{123}$ F. Würthwein, ${ }^{123}$ A. Yagil,${ }^{123}$ D. Barge, ${ }^{124}$ J. Bradmiller-Feld, ${ }^{124}$ C. Campagnari, ${ }^{124}$ T. Danielson, ${ }^{124}$ A. Dishaw, ${ }^{124}$ V. Dutta, ${ }^{124}$ K. Flowers, ${ }^{124}$ M. Franco Sevilla, ${ }^{124}$ P. Geffert, ${ }^{124}$ C. George, ${ }^{124}$ F. Golf, ${ }^{124}$ L. Gouskos, ${ }^{124}$ J. Incandela, ${ }^{124}$ C. Justus, ${ }^{124}$ N. Mccoll, ${ }^{124}$ J. Richman, ${ }^{124}$ D. Stuart, ${ }^{124}$ W. To, ${ }^{124}$ C. West, ${ }^{124}$ J. Yoo, ${ }^{124}$ A. Apresyan, ${ }^{125}$ A. Bornheim, ${ }^{125}$ J. Bunn, ${ }^{125}$ Y. Chen, ${ }^{125}$ J. Duarte, ${ }^{125}$ A. Mott, ${ }^{125}$ H. B. Newman, ${ }^{125}$ C. Pena, ${ }^{125}$ C. Rogan, ${ }^{125}$ M. Spiropulu, ${ }^{125}$ V. Timciuc, ${ }^{125}$ J. R. Vlimant, ${ }^{125}$ R. Wilkinson, ${ }^{125}$ S. Xie ${ }^{125}$ R. Y. Zhu, ${ }^{125}$ V. Azzolini, ${ }^{126}$ A. Calamba, ${ }^{126}$ B. Carlson, ${ }^{126}$ T. Ferguson, ${ }^{126}$ Y. Iiyama, ${ }^{126}$ M. Paulini, ${ }^{126}$ J. Russ, ${ }^{126}$ H. Vogel, ${ }^{126}$ I. Vorobiev, ${ }^{126}$ J. P. Cumalat, ${ }^{127}$ W. T. Ford, ${ }^{127}$ A. Gaz, ${ }^{127}$ M. Krohn, ${ }^{127}$ E. Luiggi Lopez, ${ }^{127}$ U. Nauenberg, ${ }^{127}$ J. G. Smith, ${ }^{127}$ K. Stenson, ${ }^{127}$ K. A. Ulmer, ${ }^{127}$ S. R. Wagner, ${ }^{127}$ J. Alexander, ${ }^{128}$ A. Chatterjee, ${ }^{128}$ J. Chaves, ${ }^{128}$ J. Chu, ${ }^{128}$ S. Dittmer, ${ }^{128}$ N. Eggert, ${ }^{128}$ N. Mirman, ${ }^{128}$ G. Nicolas Kaufman, ${ }^{128}$ J. R. Patterson, ${ }^{128}$ A. Ryd,${ }^{128}$ E. Salvati, ${ }^{128}$ L. Skinnari, ${ }^{128}$ W. Sun, ${ }^{128}$ W. D. Teo, ${ }^{128}$ J. Thom, ${ }^{128}$ J. Thompson, ${ }^{128}$ J. Tucker, ${ }^{128}$ Y. Weng, ${ }^{128}$ L. Winstrom, ${ }^{128}$ P. Wittich, ${ }^{128}$ D. Winn, ${ }^{129}$ S. Abdullin, ${ }^{130}$ M. Albrow, ${ }^{130}$ J. Anderson, ${ }^{130}$ G. Apollinari, ${ }^{130}$ L. A. T. Bauerdick, ${ }^{130}$ A. Beretvas, ${ }^{130}$ J. Berryhill, ${ }^{130}$ P. C. Bhat, ${ }^{130}$ G. Bolla, ${ }^{130}$ K. Burkett, ${ }^{130}$ J. N. Butler, ${ }^{130}$ H. W. K. Cheung, ${ }^{130}$ F. Chlebana, ${ }^{130}$ S. Cihangir, ${ }^{130}$ V. D. Elvira, ${ }^{130}$ I. Fisk, ${ }^{130}$ J. Freeman, ${ }^{130}$ Y. Gao, ${ }^{130}$ E. Gottschalk, ${ }^{130}$ L. Gray, ${ }^{130}$ D. Green, ${ }^{130}$ S. Grünendahl, ${ }^{130}$ O. Gutsche, ${ }^{130}$ J. Hanlon, ${ }^{130}$ 
D. Hare, ${ }^{130}$ R. M. Harris, ${ }^{130}$ J. Hirschauer, ${ }^{130}$ B. Hooberman, ${ }^{130}$ S. Jindariani, ${ }^{130}$ M. Johnson, ${ }^{130}$ U. Joshi, ${ }^{130}$ K. Kaadze, ${ }^{130}$ B. Klima,${ }^{130}$ B. Kreis, ${ }^{130}$ S. Kwan, ${ }^{130, a}$ J. Linacre, ${ }^{130}$ D. Lincoln, ${ }^{130}$ R. Lipton, ${ }^{130}$ T. Liu, ${ }^{130}$ R. Lopes De Sá, ${ }^{130}$ J. Lykken, ${ }^{130}$ K. Maeshima, ${ }^{130}$ J. M. Marraffino, ${ }^{130}$ V. I. Martinez Outschoorn, ${ }^{130}$ S. Maruyama, ${ }^{130}$ D. Mason, ${ }^{130}$ P. McBride,${ }^{130}$ P. Merkel, ${ }^{130}$ K. Mishra, ${ }^{130}$ S. Mrenna, ${ }^{130}$ Y. Musienko, ${ }^{130, d d}$ S. Nahn, ${ }^{130}$ C. Newman-Holmes, ${ }^{130}$ V. O’ Dell, ${ }^{130}$ O. Prokofyev, ${ }^{130}$ E. Sexton-Kennedy, ${ }^{130}$ S. Sharma, ${ }^{130}$ A. Soha, ${ }^{130}$ W. J. Spalding, ${ }^{130}$ L. Spiegel,,${ }^{130}$ L. Taylor, ${ }^{130}$ S. Tkaczyk, ${ }^{130}$ N. V. Tran,${ }^{130}$ L. Uplegger, ${ }^{130}$ E. W. Vaandering, ${ }^{130}$ R. Vidal, ${ }^{130}$ A. Whitbeck,,${ }^{130}$ J. Whitmore,${ }^{130}$ F. Yang, ${ }^{130}$ D. Acosta, ${ }^{131}$ P. Avery, ${ }^{131}$ P. Bortignon, ${ }^{131}$ D. Bourilkov, ${ }^{131}$ M. Carver ${ }^{131}$ D. Curry, ${ }^{131}$ S. Das, ${ }^{131}$ M. De Gruttola,${ }^{131}$ G. P. Di Giovanni, ${ }^{131}$ R. D. Field, ${ }^{131}$ M. Fisher, ${ }^{131}$ I. K. Furic, ${ }^{131}$ J. Hugon,${ }^{131}$ J. Konigsberg, ${ }^{131}$ A. Korytov, ${ }^{131}$ T. Kypreos, ${ }^{131}$ J. F. Low ${ }^{131}$ K. Matchev ${ }^{131}$ H. Mei, ${ }^{131}$ P. Milenovic, ${ }^{131, \text { aaa }}$ G. Mitselmakher ${ }^{131}$ L. Muniz, ${ }^{131}$ A. Rinkevicius,${ }^{131}$ L. Shchutska, ${ }^{131}$ M. Snowball, ${ }^{131}$ D. Sperka, ${ }^{131}$ J. Yelton, ${ }^{131}$ M. Zakaria, ${ }^{131}$ S. Hewamanage, ${ }^{132}$ S. Linn, ${ }^{132}$ P. Markowitz, ${ }^{132}$ G. Martinez, ${ }^{132}$ J. L. Rodriguez, ${ }^{132}$ T. Adams, ${ }^{133}$ A. Askew, ${ }^{133}$ J. Bochenek, ${ }^{133}$ B. Diamond, ${ }^{133}$ J. Haas, ${ }^{133}$ S. Hagopian, ${ }^{133}$ V. Hagopian, ${ }^{133}$ K. F. Johnson, ${ }^{133}$ H. Prosper, ${ }^{133}$ V. Veeraraghavan, ${ }^{133}$ M. Weinberg, ${ }^{133}$ M. M. Baarmand, ${ }^{134}$ M. Hohlmann, ${ }^{134}$ H. Kalakhety, ${ }^{134}$ F. Yumiceva, ${ }^{134}$ M. R. Adams, ${ }^{135}$ L. Apanasevich ${ }^{135}$ D. Berry, ${ }^{135}$ R. R. Betts, ${ }^{135}$ I. Bucinskaite, ${ }^{135}$ R. Cavanaugh, ${ }^{135}$ O. Evdokimov, ${ }^{135}$ L. Gauthier, ${ }^{135}$ C. E. Gerber, ${ }^{135}$ D. J. Hofman, ${ }^{135}$ P. Kurt, ${ }^{135}$ D. H. Moon, ${ }^{135}$ C. O’Brien, ${ }^{135}$ I. D. Sandoval Gonzalez, ${ }^{135}$ C. Silkworth, ${ }^{135}$ P. Turner, ${ }^{135}$ N. Varelas, ${ }^{135}$ B. Bilki, ${ }^{136, b b b}$ W. Clarida, ${ }^{136}$ K. Dilsiz, ${ }^{136}$ F. Duru, ${ }^{136}$ M. Haytmyradov, ${ }^{136}$ J.-P. Merlo, ${ }^{136}$ H. Mermerkaya, ${ }^{136, c c c}$ A. Mestvirishvili, ${ }^{136}$ A. Moeller, ${ }^{136}$ J. Nachtman, ${ }^{136}$ H. Ogul, ${ }^{136}$ Y. Onel, ${ }^{136}$ F. Ozok, ${ }^{136, \text { uu }}$ A. Penzo, ${ }^{136}$ R. Rahmat, ${ }^{136}$ S. Sen, ${ }^{136}$ P. Tan, ${ }^{136}$ E. Tiras, ${ }^{136}$ J. Wetzel, ${ }^{136}$ K. Yi ${ }^{136}$ B. A. Barnett, ${ }^{137}$ B. Blumenfeld, ${ }^{137}$ S. Bolognesi, ${ }^{137}$ D. Fehling, ${ }^{137}$ A. V. Gritsan, ${ }^{137}$ P. Maksimovic, ${ }^{137}$ C. Martin, ${ }^{137}$ M. Swartz, ${ }^{137}$ P. Baringer, ${ }^{138}$ A. Bean, ${ }^{138}$ G. Benelli, ${ }^{138}$ C. Bruner, ${ }^{138}$ R. P. Kenny III, ${ }^{138}$ M. Malek, ${ }^{138}$ M. Murray, ${ }^{138}$ D. Noonan, ${ }^{138}$ S. Sanders, ${ }^{138}$ J. Sekaric, ${ }^{138}$ R. Stringer, ${ }^{138}$ Q. Wang, ${ }^{138}$ J. S. Wood, ${ }^{138}$ I. Chakaberia, ${ }^{139}$ A. Ivanov, ${ }^{139}$ S. Khalil, ${ }^{139}$ M. Makouski, ${ }^{139}$ Y. Maravin, ${ }^{139}$ L. K. Saini, ${ }^{139}$ S. Shrestha, ${ }^{139}$ N. Skhirtladze, ${ }^{139}$ I. Svintradze, ${ }^{139}$ J. Gronberg, ${ }^{140}$ D. Lange, ${ }^{140}$ F. Rebassoo, ${ }^{140}$ D. Wright, ${ }^{140}$ A. Baden, ${ }^{141}$ A. Belloni, ${ }^{141}$ B. Calvert, ${ }^{141}$

S. C. Eno, ${ }^{141}$ J. A. Gomez, ${ }^{141}$ N. J. Hadley, ${ }^{141}$ R. G. Kellogg, ${ }^{141}$ T. Kolberg, ${ }^{141}$ Y. Lu, ${ }^{141}$ M. Marionneau, ${ }^{141}$ A. C. Mignerey, ${ }^{141}$ K. Pedro, ${ }^{141}$ A. Skuja, ${ }^{141}$ M. B. Tonjes, ${ }^{141}$ S. C. Tonwar, ${ }^{141}$ A. Apyan, ${ }^{142}$ R. Barbieri, ${ }^{142}$ G. Bauer, ${ }^{142}$ W. Busza, ${ }^{142}$ I. A. Cali, ${ }^{142}$ M. Chan, ${ }^{142}$ L. Di Matteo, ${ }^{142}$ G. Gomez Ceballos, ${ }^{142}$ M. Goncharov,${ }^{142}$ D. Gulhan,${ }^{142}$ M. Klute, ${ }^{142}$ Y. S. Lai, ${ }^{142}$ Y.-J. Lee, ${ }^{142}$ A. Levin, ${ }^{142}$ P. D. Luckey, ${ }^{142}$ T. Ma ${ }^{142}$ C. Paus, ${ }^{142}$ D. Ralph,${ }^{142}$ C. Roland, ${ }^{142}$ G. Roland, ${ }^{142}$ G. S. F. Stephans, ${ }^{142}$ F. Stöckli, ${ }^{142}$ K. Sumorok, ${ }^{142}$ D. Velicanu, ${ }^{142}$ J. Veverka, ${ }^{142}$ B. Wyslouch,${ }^{142}$ M. Yang, ${ }^{142}$ M. Zanetti, ${ }^{142}$ V. Zhukova, ${ }^{142}$ B. Dahmes, ${ }^{143}$ A. Gude ${ }^{143}$ S. C. Kao, ${ }^{143}$ K. Klapoetke, ${ }^{143}$ Y. Kubota, ${ }^{143}$ J. Mans, ${ }^{143}$ N. Pastika, ${ }^{143}$ R. Rusack, ${ }^{143}$ A. Singovsky, ${ }^{143}$ N. Tambe, ${ }^{143}$ J. Turkewitz, ${ }^{143}$ J. G. Acosta,${ }^{144}$ S. Oliveros, ${ }^{144}$ E. Avdeeva, ${ }^{145}$ K. Bloom, ${ }^{145}$ S. Bose, ${ }^{145}$ D. R. Claes, ${ }^{145}$ A. Dominguez, ${ }^{145}$ R. Gonzalez Suarez, ${ }^{145}$ J. Keller, ${ }^{145}$ D. Knowlton, ${ }^{145}$ I. Kravchenko, ${ }^{145}$ J. Lazo-Flores, ${ }^{145}$ S. Malik, ${ }^{145}$ F. Meier ${ }^{145}$ F. Ratnikov, ${ }^{145}$ G. R. Snow ${ }^{145}$ M. Zvada, ${ }^{145}$ J. Dolen, ${ }^{146}$ A. Godshalk, ${ }^{146}$ I. Iashvili, ${ }^{146}$ A. Kharchilava, ${ }^{146}$ A. Kumar, ${ }^{146}$ S. Rappoccio, ${ }^{146}$ G. Alverson, ${ }^{147}$ E. Barberis, ${ }^{147}$ D. Baumgartel, ${ }^{147}$ M. Chasco ${ }^{147}$ J. Haley, ${ }^{147}$ A. Massironi, ${ }^{147}$ D. M. Morse, ${ }^{147}$ D. Nash ${ }^{147}$ T. Orimoto, ${ }^{147}$ D. Trocino, ${ }^{147}$ R.-J. Wang, ${ }^{147}$ D. Wood, ${ }^{147}$ J. Zhang, ${ }^{147}$ K. A. Hahn, ${ }^{148}$ A. Kubik, ${ }^{148}$ N. Mucia, ${ }^{148}$ N. Odell, ${ }^{148}$ B. Pollack, ${ }^{148}$ A. Pozdnyakov, ${ }^{148}$ M. Schmitt, ${ }^{148}$ S. Stoynev, ${ }^{148}$ K. Sung, ${ }^{148}$ M. Velasco, ${ }^{148}$ S. Won, ${ }^{148}$ A. Brinkerhoff, ${ }^{149}$ K. M. Chan, ${ }^{149}$ A. Drozdetskiy, ${ }^{149}$ M. Hildreth, ${ }^{149}$ C. Jessop, ${ }^{149}$ D. J. Karmgard, ${ }^{149}$ N. Kellams, ${ }^{149}$ K. Lannon, ${ }^{149}$ S. Lynch, ${ }^{149}$ N. Marinelli, ${ }^{149}$ T. Pearson, ${ }^{149}$ M. Planer ${ }^{149}$ R. Ruchti, ${ }^{149}$ N. Valls, ${ }^{149}$ M. Wayne, ${ }^{149}$ M. Wolf, ${ }^{149}$ A. Woodard, ${ }^{149}$ L. Antonelli, ${ }^{150}$ J. Brinson, ${ }^{150}$

B. Bylsma, ${ }^{150}$ L. S. Durkin, ${ }^{150}$ S. Flowers, ${ }^{150}$ A. Hart,${ }^{150}$ C. Hill, ${ }^{150}$ R. Hughes,${ }^{150}$ K. Kotov, ${ }^{150}$ T. Y. Ling, ${ }^{150}$ W. Luo, ${ }^{150}$ D. Puigh, ${ }^{150}$ M. Rodenburg, ${ }^{150}$ G. Smith, ${ }^{150}$ B. L. Winer ${ }^{150}$ H. Wolfe ${ }^{150}$ H. W. Wulsin, ${ }^{150}$ O. Driga ${ }^{151}$ P. Elmer, ${ }^{151}$ J. Hardenbrook ${ }^{151}$ P. Hebda ${ }^{151}$ A. Hunt, ${ }^{151}$ S. A. Koay,${ }^{151}$ P. Lujan, ${ }^{151}$ D. Marlow, ${ }^{151}$ T. Medvedeva, ${ }^{151}$ M. Mooney, ${ }^{151}$ J. Olsen, ${ }^{151}$ P. Piroué, ${ }^{151}$ X. Quan, ${ }^{151}$ H. Saka, ${ }^{151}$ D. Stickland, ${ }^{151, c}$ C. Tully, ${ }^{151}$ J. S. Werner, ${ }^{151}$ A. Zuranski, ${ }^{151}$

E. Brownson, ${ }^{152}$ H. Mendez, ${ }^{152}$ J. E. Ramirez Vargas, ${ }^{152}$ V. E. Barnes, ${ }^{153}$ D. Benedetti, ${ }^{153}$ D. Bortoletto, ${ }^{153}$ M. De Mattia, ${ }^{153}$

L. Gutay, ${ }^{153}$ Z. Hu, ${ }^{153}$ M. K. Jha, ${ }^{153}$ M. Jones, ${ }^{153}$ K. Jung, ${ }^{153}$ M. Kress, ${ }^{153}$ N. Leonardo, ${ }^{153}$ D. Lopes Pegna, ${ }^{153}$

V. Maroussov, ${ }^{153}$ D. H. Miller, ${ }^{153}$ N. Neumeister, ${ }^{153}$ B. C. Radburn-Smith, ${ }^{153}$ X. Shi, ${ }^{153}$ I. Shipsey, ${ }^{153}$ D. Silvers, ${ }^{153}$ A. Svyatkovskiy, ${ }^{153}$ F. Wang, ${ }^{153}$ W. Xie, ${ }^{153}$ L. Xu,${ }^{153}$ H. D. Yoo,${ }^{153}$ J. Zablocki, ${ }^{153}$ Y. Zheng, ${ }^{153}$ N. Parashar, ${ }^{154}$ J. Stupak,${ }^{154}$ A. Adair, ${ }^{155}$ B. Akgun, ${ }^{155}$ K. M. Ecklund ${ }^{155}$ F. J. M. Geurts, ${ }^{155}$ W. Li,${ }^{155}$ B. Michlin, ${ }^{155}$ B. P. Padley, ${ }^{155}$ R. Redjimi, ${ }^{155}$ J. Roberts, ${ }^{155}$ J. Zabel, ${ }^{155}$ B. Betchart, ${ }^{156}$ A. Bodek,${ }^{156}$ R. Covarelli, ${ }^{156}$ P. de Barbaro, ${ }^{156}$ R. Demina, ${ }^{156}$ Y. Eshaq, ${ }^{156}$ T. Ferbel, ${ }^{156}$ A. Garcia-Bellido, ${ }^{156}$ P. Goldenzweig, ${ }^{156}$ J. Han,${ }^{156}$ A. Harel, ${ }^{156}$ A. Khukhunaishvili, ${ }^{156}$ S. Korjenevski, ${ }^{156}$ G. Petrillo, ${ }^{156}$ D. Vishnevskiy, ${ }^{156}$ R. Ciesielski, ${ }^{157}$ L. Demortier, ${ }^{157}$ K. Goulianos, ${ }^{157}$ G. Lungu, ${ }^{157}$ C. Mesropian, ${ }^{157}$ 
S. Arora, ${ }^{158}$ A. Barker, ${ }^{158}$ J. P. Chou, ${ }^{158}$ C. Contreras-Campana, ${ }^{158}$ E. Contreras-Campana, ${ }^{158}$ D. Duggan, ${ }^{158}$ D. Ferencek, ${ }^{158}$ Y. Gershtein, ${ }^{158}$ R. Gray, ${ }^{158}$ E. Halkiadakis, ${ }^{158}$ D. Hidas, ${ }^{158}$ S. Kaplan, ${ }^{158}$ A. Lath,${ }^{158}$ S. Panwalkar, ${ }^{158}$ M. Park, ${ }^{158}$ R. Patel,${ }^{158}$ S. Salur, ${ }^{158}$ S. Schnetzer, ${ }^{158}$ S. Somalwar, ${ }^{158}$ R. Stone, ${ }^{158}$ S. Thomas, ${ }^{158}$ P. Thomassen, ${ }^{158}$ M. Walker, ${ }^{158}$ K. Rose, ${ }^{159}$ S. Spanier ${ }^{159}$ A. York, ${ }^{159}$ O. Bouhali, ${ }^{160, \text { ddd }}$ A. Castaneda Hernandez,${ }^{160}$ R. Eusebi, ${ }^{160}$ W. Flanagan, ${ }^{160}$ J. Gilmore, ${ }^{160}$ T. Kamon, ${ }^{160, \text { eee }}$ V. Khotilovich, ${ }^{160}$ V. Krutelyov, ${ }^{160}$ R. Montalvo, ${ }^{160}$ I. Osipenkov, ${ }^{160}$ Y. Pakhotin, ${ }^{160}$ A. Perloff,,${ }^{160}$ J. Roe, ${ }^{160}$ A. Rose ${ }^{160}$ A. Safonov, ${ }^{160}$ I. Suarez, ${ }^{160}$ A. Tatarinov, ${ }^{160}$ N. Akchurin, ${ }^{161}$ C. Cowden, ${ }^{161}$ J. Damgov, ${ }^{161}$ C. Dragoiu, ${ }^{161}$ P. R. Dudero, ${ }^{161}$ J. Faulkner, ${ }^{161}$ K. Kovitanggoon, ${ }^{161}$ S. Kunori, ${ }^{161}$ S. W. Lee, ${ }^{161}$ T. Libeiro, ${ }^{161}$ I. Volobouev, ${ }^{161}$ E. Appelt, ${ }^{162}$ A. G. Delannoy, ${ }^{162}$ S. Greene, ${ }^{162}$ A. Gurrola, ${ }^{162}$ W. Johns, ${ }^{162}$ C. Maguire, ${ }^{162}$ Y. Mao, ${ }^{162}$ A. Melo, ${ }^{162}$ M. Sharma, ${ }^{162}$ P. Sheldon, ${ }^{162}$ B. Snook, ${ }^{162}$ S. Tuo, ${ }^{162}$ J. Velkovska, ${ }^{162}$ M. W. Arenton, ${ }^{163}$ S. Boutle, ${ }^{163}$ B. Cox, ${ }^{163}$ B. Francis, ${ }^{163}$ J. Goodell, ${ }^{163}$ R. Hirosky, ${ }^{163}$ A. Ledovskoy, ${ }^{163}$ H. Li, ${ }^{163}$ C. Lin, ${ }^{163}$ C. Neu, ${ }^{163}$ J. Wood, ${ }^{163}$ C. Clarke, ${ }^{164}$ R. Harr, ${ }^{164}$ P. E. Karchin, ${ }^{164}$ C. Kottachchi Kankanamge Don, ${ }^{164}$ P. Lamichhane, ${ }^{164}$ J. Sturdy, ${ }^{164}$ D. A. Belknap, ${ }^{165}$ D. Carlsmith, ${ }^{165}$ M. Cepeda, ${ }^{165}$ S. Dasu, ${ }^{165}$ L. Dodd, ${ }^{165}$ S. Duric, ${ }^{165}$ E. Friis, ${ }^{165}$ R. Hall-Wilton, ${ }^{165}$ M. Herndon, ${ }^{165}$ A. Hervé, ${ }^{165}$ P. Klabbers, ${ }^{165}$ A. Lanaro, ${ }^{165}$ C. Lazaridis, ${ }^{165}$ A. Levine, ${ }^{165}$ R. Loveless, ${ }^{165}$ A. Mohapatra, ${ }^{165}$ I. Ojalvo, ${ }^{165}$ T. Perry, ${ }^{165}$ G. A. Pierro, ${ }^{165}$ G. Polese, ${ }^{165}$ I. Ross, ${ }^{165}$ T. Sarangi, ${ }^{165}$ A. Savin, ${ }^{165}$ W. H. Smith, ${ }^{165}$ D. Taylor, ${ }^{165}$ P. Verwilligen, ${ }^{165}$ C. Vuosalo, ${ }^{165}$ and N. Woods ${ }^{165}$

\title{
(CMS Collaboration)
}

\author{
${ }^{1}$ Yerevan Physics Institute, Yerevan, Armenia \\ ${ }^{2}$ Institut für Hochenergiephysik der OeAW, Wien, Austria \\ ${ }^{3}$ National Centre for Particle and High Energy Physics, Minsk, Belarus \\ ${ }^{4}$ Universiteit Antwerpen, Antwerpen, Belgium \\ ${ }^{5}$ Vrije Universiteit Brussel, Brussel, Belgium \\ ${ }^{6}$ Université Libre de Bruxelles, Bruxelles, Belgium \\ ${ }^{7}$ Ghent University, Ghent, Belgium \\ ${ }^{8}$ Université Catholique de Louvain, Louvain-la-Neuve, Belgium \\ ${ }^{9}$ Université de Mons, Mons, Belgium \\ ${ }^{10}$ Centro Brasileiro de Pesquisas Fisicas, Rio de Janeiro, Brazil \\ ${ }^{11}$ Universidade do Estado do Rio de Janeiro, Rio de Janeiro, Brazil \\ ${ }^{12 a}$ Universidade Estadual Paulista, São Paulo, Brazil \\ ${ }^{12 \mathrm{~b}}$ Universidade Federal do ABC, São Paulo, Brazil \\ ${ }^{13}$ Institute for Nuclear Research and Nuclear Energy, Sofia, Bulgaria \\ ${ }^{14}$ University of Sofia, Sofia, Bulgaria \\ ${ }^{15}$ Institute of High Energy Physics, Beijing, China \\ ${ }^{16}$ State Key Laboratory of Nuclear Physics and Technology, Peking University, Beijing, China \\ ${ }^{17}$ Universidad de Los Andes, Bogota, Colombia \\ ${ }^{18}$ University of Split, Faculty of Electrical Engineering, Mechanical Engineering and Naval Architecture, Split, Croatia \\ ${ }^{19}$ University of Split, Faculty of Science, Split, Croatia \\ ${ }^{20}$ Institute Rudjer Boskovic, Zagreb, Croatia \\ ${ }^{21}$ University of Cyprus, Nicosia, Cyprus \\ ${ }^{22}$ Charles University, Prague, Czech Republic \\ ${ }^{23}$ Academy of Scientific Research and Technology of the Arab Republic of Egypt, Egyptian Network of High Energy Physics, \\ Cairo, Egypt \\ ${ }^{24}$ National Institute of Chemical Physics and Biophysics, Tallinn, Estonia \\ ${ }^{25}$ Department of Physics, University of Helsinki, Helsinki, Finland \\ ${ }^{26}$ Helsinki Institute of Physics, Helsinki, Finland \\ ${ }^{27}$ Lappeenranta University of Technology, Lappeenranta, Finland \\ ${ }^{28}$ DSM/IRFU, CEA/Saclay, Gif-sur-Yvette, France \\ ${ }^{29}$ Laboratoire Leprince-Ringuet, Ecole Polytechnique, IN2P3-CNRS, Palaiseau, France \\ ${ }^{30}$ Institut Pluridisciplinaire Hubert Curien, Université de Strasbourg, Université de Haute Alsace Mulhouse, \\ CNRS/IN2P3, Strasbourg, France \\ ${ }^{31}$ Centre de Calcul de l'Institut National de Physique Nucleaire et de Physique des Particules, CNRS/IN2P3, Villeurbanne, France \\ ${ }^{32}$ Université de Lyon, Université Claude Bernard Lyon 1, CNRS-IN2P3, Institut de Physique Nucléaire de Lyon, Villeurbanne, France \\ ${ }^{33}$ Institute of High Energy Physics and Informatization, Tbilisi State University, Tbilisi, Georgia \\ ${ }^{34}$ RWTH Aachen University, I. Physikalisches Institut, Aachen, Germany \\ ${ }^{35}$ RWTH Aachen University, III. Physikalisches Institut A, Aachen, Germany
}


${ }^{36}$ RWTH Aachen University, III. Physikalisches Institut B, Aachen, Germany

${ }^{37}$ Deutsches Elektronen-Synchrotron, Hamburg, Germany

${ }^{38}$ University of Hamburg, Hamburg, Germany

${ }^{39}$ Institut für Experimentelle Kernphysik, Karlsruhe, Germany

${ }^{40}$ Institute of Nuclear and Particle Physics (INPP), NCSR Demokritos, Aghia Paraskevi, Greece

${ }^{41}$ University of Athens, Athens, Greece

${ }^{42}$ University of Ioánnina, Ioánnina, Greece

${ }^{43}$ Wigner Research Centre for Physics, Budapest, Hungary

${ }^{44}$ Institute of Nuclear Research ATOMKI, Debrecen, Hungary

${ }^{45}$ University of Debrecen, Debrecen, Hungary

${ }^{46}$ National Institute of Science Education and Research, Bhubaneswar, India

${ }^{47}$ Panjab University, Chandigarh, India

${ }^{48}$ University of Delhi, Delhi, India

${ }^{49}$ Saha Institute of Nuclear Physics, Kolkata, India

${ }^{50}$ Bhabha Atomic Research Centre, Mumbai, India

${ }^{51}$ Tata Institute of Fundamental Research, Mumbai, India

${ }^{52}$ Institute for Research in Fundamental Sciences (IPM), Tehran, Iran

${ }^{53}$ University College Dublin, Dublin, Ireland

${ }^{54 a}$ INFN Sezione di Bari, Bari, Italy

${ }^{54 \mathrm{~b}}$ Università di Bari, Bari, Italy

${ }^{54 \mathrm{c}}$ Politecnico di Bari, Bari, Italy

${ }^{55 a}$ INFN Sezione di Bologna, Bologna, Italy

${ }^{55 \mathrm{~b}}$ Università di Bologna, Bologna, Italy

${ }^{56 a}$ INFN Sezione di Catania, Catania, Italy

${ }^{56 \mathrm{~b}}$ Università di Catania, Catania, Italy

${ }^{56 c}$ CSFNSM, Catania, Italy

${ }^{57 a}$ INFN Sezione di Firenze, Firenze, Italy

${ }^{57 \mathrm{~b}}$ Università di Firenze, Firenze, Italy

${ }^{58}$ INFN Laboratori Nazionali di Frascati, Frascati, Italy

${ }^{59 a}$ INFN Sezione di Genova, Genova, Italy

${ }^{59 \mathrm{~b}}$ Università di Genova, Genova, Italy

${ }^{60 a}$ INFN Sezione di Milano-Bicocca, Milano, Italy

${ }^{60 \mathrm{~b}}$ Università di Milano-Bicocca, Milano, Italy

${ }^{61 a}$ INFN Sezione di Napoli, Napoli, Italy

${ }^{61 \mathrm{~b}}$ Università di Napoli 'Federico II', Napoli, Italy

${ }^{61 \mathrm{c}}$ Università della Basilicata (Potenza), Napoli, Italy

${ }^{61 \mathrm{~d} U n i v e r s i t a ̀ ~ G u g l i e l m o ~ M a r c o n i ~(R o m a), ~ N a p o l i, ~ I t a l y ~}$

${ }^{62 a}$ INFN Sezione di Padova, Padova, Italy

${ }^{62 \mathrm{~b}}$ Università di Padova, Padova, Italy

${ }^{62 \mathrm{c}}$ Università di Trento (Trento), Padova, Italy

${ }^{63 a}$ INFN Sezione di Pavia, Pavia, Italy

${ }^{63 \mathrm{~b}}$ Università di Pavia, Pavia, Italy

${ }^{64 a}$ INFN Sezione di Perugia, Perugia, Italy

${ }^{64 \mathrm{~b}}$ Università di Perugia, Perugia, Italy

${ }^{65 a}$ INFN Sezione di Pisa, Pisa, Italy

${ }^{65 \mathrm{~b}}$ Università di Pisa, Pisa, Italy

${ }^{65 \mathrm{c}}$ Scuola Normale Superiore di Pisa, Pisa, Italy

${ }^{66 a}$ INFN Sezione di Roma, Roma, Italy

${ }^{66 \mathrm{~b}}$ Università di Roma, Roma, Italy

${ }^{67 a}$ INFN Sezione di Torino, Torino, Italy

${ }^{67 \mathrm{~b}}$ Università di Torino, Torino, Italy

${ }^{67 \mathrm{c}}$ Università del Piemonte Orientale (Novara), Torino, Italy

${ }^{68 \mathrm{a}}$ INFN Sezione di Trieste, Trieste, Italy

${ }^{68 \mathrm{~b}}$ Università di Trieste, Trieste, Italy

${ }^{69}$ Kangwon National University, Chunchon, Korea

${ }^{70}$ Kyungpook National University, Daegu, Korea

${ }^{71}$ Chonbuk National University, Jeonju, Korea

${ }^{72}$ Chonnam National University, Institute for Universe and Elementary Particles, Kwangju, Korea

${ }^{73}$ Korea University, Seoul, Korea

${ }^{74}$ University of Seoul, Seoul, Korea 
${ }^{75}$ Sungkyunkwan University, Suwon, Korea

${ }^{76}$ Vilnius University, Vilnius, Lithuania

${ }^{77}$ National Centre for Particle Physics, Universiti Malaya, Kuala Lumpur, Malaysia

${ }^{78}$ Centro de Investigacion y de Estudios Avanzados del IPN, Mexico City, Mexico

${ }^{79}$ Universidad Iberoamericana, Mexico City, Mexico

${ }^{80}$ Benemerita Universidad Autonoma de Puebla, Puebla, Mexico

${ }^{81}$ Universidad Autónoma de San Luis Potosí, San Luis Potosí, Mexico

${ }^{82}$ University of Auckland, Auckland, New Zealand

${ }^{83}$ University of Canterbury, Christchurch, New Zealand

${ }^{84}$ National Centre for Physics, Quaid-I-Azam University, Islamabad, Pakistan

${ }^{85}$ National Centre for Nuclear Research, Swierk, Poland

${ }^{86}$ Institute of Experimental Physics, Faculty of Physics, University of Warsaw, Warsaw, Poland

${ }^{87}$ Laboratório de Instrumentação e Física Experimental de Partículas, Lisboa, Portugal

${ }^{88}$ Joint Institute for Nuclear Research, Dubna, Russia

${ }^{89}$ Petersburg Nuclear Physics Institute, Gatchina (St. Petersburg), Russia

${ }^{90}$ Institute for Nuclear Research, Moscow, Russia

${ }^{91}$ Institute for Theoretical and Experimental Physics, Moscow, Russia

${ }^{92}$ P.N. Lebedev Physical Institute, Moscow, Russia

${ }^{93}$ Skobeltsyn Institute of Nuclear Physics, Lomonosov Moscow State University, Moscow, Russia

${ }^{94}$ State Research Center of Russian Federation, Institute for High Energy Physics, Protvino, Russia

${ }^{95}$ University of Belgrade, Faculty of Physics and Vinca Institute of Nuclear Sciences, Belgrade, Serbia

${ }^{96}$ Centro de Investigaciones Energéticas Medioambientales y Tecnológicas (CIEMAT), Madrid, Spain

${ }^{97}$ Universidad Autónoma de Madrid, Madrid, Spain

${ }^{98}$ Universidad de Oviedo, Oviedo, Spain

${ }^{99}$ Instituto de Física de Cantabria (IFCA), CSIC-Universidad de Cantabria, Santander, Spain

${ }^{100}$ CERN, European Organization for Nuclear Research, Geneva, Switzerland

${ }^{101}$ Paul Scherrer Institut, Villigen, Switzerland

${ }^{102}$ Institute for Particle Physics, ETH Zurich, Zurich, Switzerland

${ }^{103}$ Universität Zürich, Zurich, Switzerland

${ }^{104}$ National Central University, Chung-Li, Taiwan

${ }^{105}$ National Taiwan University (NTU), Taipei, Taiwan

${ }^{106}$ Chulalongkorn University, Faculty of Science, Department of Physics, Bangkok, Thailand

${ }^{107}$ Cukurova University, Adana, Turkey

${ }^{108}$ Middle East Technical University, Physics Department, Ankara, Turkey

${ }^{109}$ Bogazici University, Istanbul, Turkey

${ }^{110}$ Istanbul Technical University, Istanbul, Turkey

${ }^{111}$ National Scientific Center, Kharkov Institute of Physics and Technology, Kharkov, Ukraine

${ }^{112}$ University of Bristol, Bristol, United Kingdom

${ }^{113}$ Rutherford Appleton Laboratory, Didcot, United Kingdom

${ }^{114}$ Imperial College, London, United Kingdom

${ }^{115}$ Brunel University, Uxbridge, United Kingdom

${ }^{116}$ Baylor University, Waco, USA

${ }^{117}$ The University of Alabama, Tuscaloosa, USA

${ }^{118}$ Boston University, Boston, USA

${ }^{119}$ Brown University, Providence, USA

${ }^{120}$ University of California, Davis, Davis, USA

${ }^{121}$ University of California, Los Angeles, USA

${ }^{122}$ University of California, Riverside, Riverside, USA

${ }^{123}$ University of California, San Diego, La Jolla, USA

${ }^{124}$ University of California, Santa Barbara, Santa Barbara, USA

${ }^{125}$ California Institute of Technology, Pasadena, USA

${ }^{126}$ Carnegie Mellon University, Pittsburgh, USA

${ }^{127}$ University of Colorado at Boulder, Boulder, USA

${ }^{128}$ Cornell University, Ithaca, USA

${ }^{129}$ Fairfield University, Fairfield, USA

${ }^{130}$ Fermi National Accelerator Laboratory, Batavia, USA

${ }^{131}$ University of Florida, Gainesville, USA

${ }^{132}$ Florida International University, Miami, USA

${ }^{133}$ Florida State University, Tallahassee, USA

${ }^{134}$ Florida Institute of Technology, Melbourne, USA 
${ }^{135}$ University of Illinois at Chicago (UIC), Chicago, USA

${ }^{136}$ The University of Iowa, Iowa City, USA

${ }^{137}$ Johns Hopkins University, Baltimore, USA

${ }^{138}$ The University of Kansas, Lawrence, USA

${ }^{139}$ Kansas State University, Manhattan, USA

${ }^{140}$ Lawrence Livermore National Laboratory, Livermore, USA

${ }^{141}$ University of Maryland, College Park, USA

${ }^{142}$ Massachusetts Institute of Technology, Cambridge, USA

${ }^{143}$ University of Minnesota, Minneapolis, USA

${ }^{144}$ University of Mississippi, Oxford, USA

${ }^{145}$ University of Nebraska-Lincoln, Lincoln, USA

${ }^{146}$ State University of New York at Buffalo, Buffalo, USA

${ }^{147}$ Northeastern University, Boston, USA

${ }^{148}$ Northwestern University, Evanston, USA

${ }^{149}$ University of Notre Dame, Notre Dame, USA

${ }^{150}$ The Ohio State University, Columbus, USA

${ }^{151}$ Princeton University, Princeton, USA

${ }^{152}$ University of Puerto Rico, Mayaguez, USA

${ }^{153}$ Purdue University, West Lafayette, USA

${ }^{154}$ Purdue University Calumet, Hammond, USA

${ }^{155}$ Rice University, Houston, USA

${ }^{156}$ University of Rochester, Rochester, USA

${ }^{157}$ The Rockefeller University, New York, USA

${ }^{158}$ Rutgers, The State University of New Jersey, Piscataway, USA

${ }^{159}$ University of Tennessee, Knoxville, USA

${ }^{160}$ Texas A\&M University, College Station, USA

${ }^{161}$ Texas Tech University, Lubbock, USA

${ }^{162}$ Vanderbilt University, Nashville, USA

${ }^{163}$ University of Virginia, Charlottesville, USA

${ }^{164}$ Wayne State University, Detroit, USA

${ }^{165}$ University of Wisconsin, Madison, USA

${ }^{\mathrm{a}}$ Deceased.

${ }^{\mathrm{b}}$ Also at Vienna University of Technology, Vienna, Austria.

${ }^{c}$ Also at CERN, European Organization for Nuclear Research, Geneva, Switzerland.

${ }^{\mathrm{d}}$ Also at Institut Pluridisciplinaire Hubert Curien, Université de Strasbourg, Université de Haute Alsace Mulhouse, CNRS/IN2P3, Strasbourg, France.

${ }^{\mathrm{e}}$ Also at National Institute of Chemical Physics and Biophysics, Tallinn, Estonia.

${ }^{\mathrm{f}}$ Also at Skobeltsyn Institute of Nuclear Physics, Lomonosov Moscow State University, Moscow, Russia.

${ }^{\mathrm{g}}$ Also at Universidade Estadual de Campinas, Campinas, Brazil.

${ }^{\mathrm{h}}$ Also at Laboratoire Leprince-Ringuet, Ecole Polytechnique, IN2P3-CNRS, Palaiseau, France.

${ }^{\mathrm{i}}$ Also at Joint Institute for Nuclear Research, Dubna, Russia.

${ }^{\mathrm{j}}$ Also at Suez University, Suez, Egypt.

${ }^{\mathrm{k}}$ Also at Cairo University, Cairo, Egypt.

${ }^{1}$ Also at Fayoum University, El-Fayoum, Egypt.

${ }^{\mathrm{m}}$ Also at British University in Egypt, Cairo, Egypt.

${ }^{\mathrm{n}}$ Also at Sultan Qaboos University, Muscat, Oman.

${ }^{\circ}$ Also at Université de Haute Alsace, Mulhouse, France.

${ }^{\mathrm{p}}$ Also at Brandenburg University of Technology, Cottbus, Germany.

${ }^{\mathrm{q}}$ Also at Institute of Nuclear Research ATOMKI, Debrecen, Hungary.

${ }^{\mathrm{r}}$ Also at Eötvös Loránd University, Budapest, Hungary.

${ }^{\mathrm{s}}$ Also at University of Debrecen, Debrecen, Hungary.

${ }^{t}$ Also at University of Visva-Bharati, Santiniketan, India.

"Also at King Abdulaziz University, Jeddah, Saudi Arabia.

${ }^{v}$ Also at University of Ruhuna, Matara, Sri Lanka.

${ }^{\mathrm{w}}$ Also at Isfahan University of Technology, Isfahan, Iran.

${ }^{x}$ Also at University of Tehran, Department of Engineering Science, Tehran, Iran.

${ }^{y}$ Also at Plasma Physics Research Center, Science and Research Branch, Islamic Azad University, Tehran, Iran.

${ }^{\mathrm{z}}$ Also at Università degli Studi di Siena, Siena, Italy.

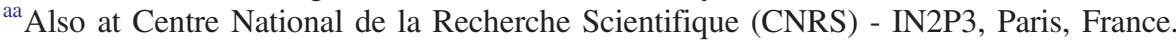


${ }^{\mathrm{bb}}$ Also at Purdue University, West Lafayette, USA.

${ }^{c c}$ Also at Universidad Michoacana de San Nicolas de Hidalgo, Morelia, Mexico.

${ }^{\mathrm{dd}}$ Also at Institute for Nuclear Research, Moscow, Russia.

${ }^{\mathrm{ee}}$ Also at St. Petersburg State Polytechnical University, St. Petersburg, Russia.

${ }^{\mathrm{ff}}$ Also at California Institute of Technology, Pasadena, USA.

${ }^{g g}$ Also at Faculty of Physics, University of Belgrade, Belgrade, Serbia.

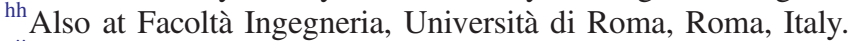

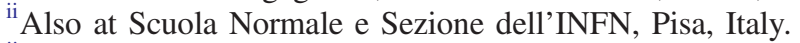

${ }^{\mathrm{jj}}$ Also at University of Athens, Athens, Greece.

${ }^{\mathrm{kk}}$ Also at Paul Scherrer Institut, Villigen, Switzerland.

${ }^{11}$ Also at Institute for Theoretical and Experimental Physics, Moscow, Russia.

${ }^{\mathrm{mm}}$ Also at Albert Einstein Center for Fundamental Physics, Bern, Switzerland.

${ }^{n n}$ Also at Gaziosmanpasa University, Tokat, Turkey.

${ }^{\circ o}$ Also at Adiyaman University, Adiyaman, Turkey.

${ }^{\mathrm{pp}}$ Also at Cag University, Mersin, Turkey.

${ }^{\mathrm{qq}}$ Also at Anadolu University, Eskisehir, Turkey.

${ }^{\mathrm{rr}}$ Also at Ozyegin University, Istanbul, Turkey.

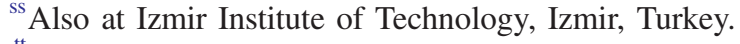

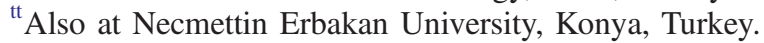

${ }^{u u}$ Also at Mimar Sinan University, Istanbul, Istanbul, Turkey.

${ }^{\mathrm{vv}}$ Also at Marmara University, Istanbul, Turkey.

${ }^{w w}$ Also at Kafkas University, Kars, Turkey.

${ }^{x x}$ Also at Yildiz Technical University, Istanbul, Turkey.

${ }^{y y}$ Also at Rutherford Appleton Laboratory, Didcot, United Kingdom.

${ }^{z z}$ Also at School of Physics and Astronomy, University of Southampton, Southampton, United Kingdom.

${ }^{\text {aaa }}$ Also at University of Belgrade, Faculty of Physics and Vinca Institute of Nuclear Sciences, Belgrade, Serbia.

${ }^{\mathrm{bbb}}$ Also at Argonne National Laboratory, Argonne, USA.

${ }^{\mathrm{ccc}}$ Also at Erzincan University, Erzincan, Turkey.

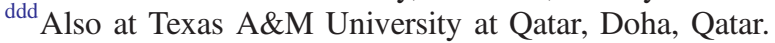

${ }^{e e e}$ Also at Kyungpook National University, Daegu, Korea. 